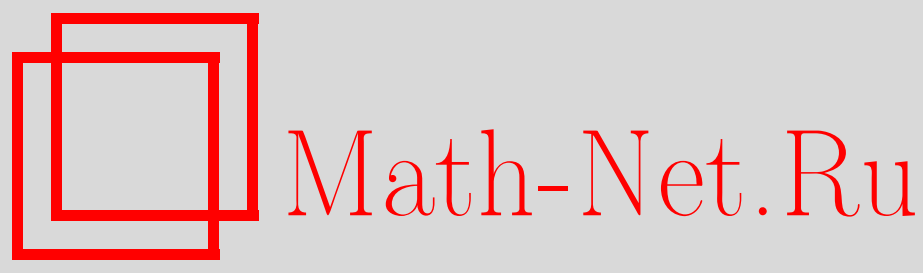

В. В. Пупышев, Метод амплитудных функций в теории двумерного рассеяния, ТМФ, 2017, том 191, номер 1, 3462

DOI: https://doi.org/10.4213/tmf9162

Использование Общероссийского математического портала Math-Net.Ru подразумевает, что вы прочитали и согласны с пользовательским соглашением http://www.mathnet.ru/rus/agreement

Параметры загрузки:

IP : 34.229 .45 .116

26 апреля 2023 г., 16:03:14

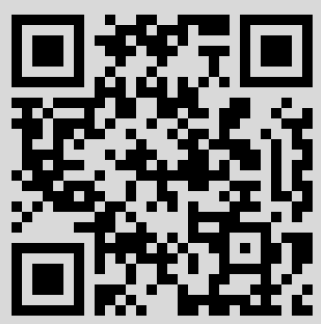




\title{
МЕТОД АМПЛИТУДНЫХ ФУНКЦИЙ В ТЕОРИИ ДВУМЕРНОГО РАССЕЯНИЯ
}

\begin{abstract}
Даны формулировка и математическое обоснование метода амплитудных функций, предназначенного для решения радиальной задачи двумерного рассеяния квантовой частицы суммой кулоновского потенциала и некоторого центрального коротко- или дальнодействующего потенциала.
\end{abstract}

Ключевые слова: двумерное рассеяние, суперпозиция кулоновского и центрального коротко- или дальнодействующего потенциалов, метод фазовых функций.

DOI: https://doi.org/10.4213/tmf9162

\section{1. ВВЕДЕНИЕ}

В настоящее время интенсивно развивается теоретическая и экспериментальная физика ультрахолодных атомов и молекул, движущихся в магнитооптических ловушках [1], на плоских металлических поверхностях [2] и на плоских пленках из жидкого гелия [3]-[5]. Достигнутый уровень экспериментальной техники настолько высок, что в последние годы стало возможным исследовать ион-атомные столкновения в ограниченной геометрии магнитооптических ловушек. Обширный список литературы по таким столкновениям содержит недавняя работа [6], в которой впервые дан квантово-механический анализ резонансного рассеяния атома на ионе в ловушке.

Квантовое движение частиц ультрахолодных атомов или молекул в дискообразной магнитооптической ловушке, как и на плоских металлических пластинах или на плоских пленках из жидкого гелия, является квазидвумерным в следующем смысле. Атомы или молекулы остаются трехмерными (объемными) объектами. Все парные кулоновские взаимодействия между электронами и ядрами, приводящие к образованию атомов или молекул, являются такими же, как и в трехмерном пространстве, но центры масс атомов или молекул движутся в одной плоскости. Плотность ультрахолодных ансамблей атомов или молекул настолько мала, что в данной плоскости наиболее вероятными оказываются парные столкновения их частиц. В эффективно двухчастичной модели квазидвумерное столкновение двух частиц в лабораторной

* Объединенный институт ядерных исследований, Дубна, Московская обл., Россия. E-mail: pupyshev@theor.jinr.ru 
системе, связанной с одной из частиц, описывается задачей двумерного рассеяния одной квантовой частицы неподвижным силовым центром, воздействующим на нее посредством некоторого эффективного потенциала. Построение теории двумерного рассеяния подразумевает решение такой задачи для всех возможных типов эффективных потенциалов. К ним относятся центральный коротко- или дальнодействующий потенциал, кулоновский потенциал, а также суммы таких потенциалов.

Двумерное рассеяние квантовой частицы центральным короткодействующим потенциалом исследовалось многими авторами. Полученные результаты пояснены и просуммированы в классической монографии [7], посвященной методу фазовых функций в квантовой механике, и в современном курсе теории рассеяния [8]. Полный анализ двумерного рассеяния квантовой частицы центральным короткодействующим потенциалом в пределе ее низких энергий дан в работах [9]-[12].

Задача рассеяния квантовой частицы кулоновским потенциалом в двумерном пространстве имеет точное решение [13]. Многие свойства этого решения и всех порожденных им кулоновских функций выявлены в давно известных фундаментальных работах [14], [15] и в недавних статьях [16], [17].

Двумерное рассеяние квантовой частицы суммой кулоновского потенциала и некоторого центрального потенциала, принадлежащего довольно широкому классу коротко- или дальнодействующих потенциалов, еще не исследовалось. Для исследования такого рассеяния прежде всего необходимо предложить простой, но математически обоснованный и физически прозрачный подход. Главная цель настоящей работы - показать, что именно таким подходом является метод амплитудных функций. В разделе 2 мы поясняем постановку исходной задачи. Разделы 3,4 и 5 посвящены соответственно формулировке, математическому обоснованию и приложениям предлагаемого метода. Итоги выполненных исследований подведены в заключении.

\section{2. ПОСТАНОВКА ИСХОДНОЙ ЗАДАЧИ}

Объект нашего исследования - упругое рассеяние квантовой частицы $p_{1}$ в двумерной плоскости $\mathcal{P}$ ее трехмерного координатного пространства неподвижным силовым центром $O$, лежащим в той же плоскости и воздействующим на эту частицу посредством суперпозиции $V^{\mathrm{c}}+\widetilde{V}$ кулоновского потенциала $V^{\mathrm{c}}$ и некоторого центрального потенциала $\widetilde{V}$.

В плоскости $\mathcal{P}$ введем стандартным образом полярную систему координат с начальной точкой, совпадающей с силовым центром $O$. Положение квантовой частицы $p_{1}$ в плоскости $\mathcal{P}$ определим ее полярными координатами $\mathbf{r}=(r, \varphi)$. Символами $m_{1}, z_{1}, E$ и $\mathbf{k}$ обозначим массу, кулоновский заряд, полную энергию и волновой вектор этой частицы. Мы считаем, что волновой вектор $\mathbf{k}_{0}$ ее начального состояния имеет нулевой азимутальный угол $(\varphi=0)$, а силовой центр $O$ обладает кулоновским зарядом $Z$. По определению оба заряда $z_{1}$ и $Z$ кратны заряду электрона.

Пусть $\hbar-$ постоянная Планка. Положим

$$
\begin{gathered}
k=|\mathbf{k}| \equiv \sqrt{\frac{2 m_{1} E}{\hbar^{2}}}, \quad R \equiv \frac{\hbar^{2}}{2 m_{1} z_{1} Z}, \quad q=k|R|, \quad \eta \equiv \frac{1}{2 k R}=\frac{\operatorname{sgn} R}{2 q} ; \\
x \equiv \frac{r}{|R|}, \quad \rho \equiv k r=q x, \quad x=2 \rho|\eta| .
\end{gathered}
$$


Здесь и далее $k$ и $q$ - размерное и безразмерное волновые числа частицы $p_{1}, \eta-$ параметр Зоммерфельда, $x$ и $\rho$ - безразмерные неотрицательные переменные.

Кулоновский потенциал $V^{\mathrm{c}}$ определим формулой $V^{\mathrm{c}}(r)=z_{1} Z / r$. Такому потенциалу отвечают размерный и безразмерные потенциалы $1 / R r$ и $2 \eta / \rho$.

Сформулируем ограничения на исходный потенциал $\widetilde{V}$. Пусть функция $\widetilde{V}(r)$ принадлежит пространству $\mathcal{C}_{(0, \infty)}^{0}$ непрерывных на интервале $0<r<\infty$ функций и удовлетворяет условию $\widetilde{V}(r)=o\left(r^{-2}\right)$ при $r \rightarrow 0$ и при $r \rightarrow \infty$. Вследствие этих ограничений при любом конечном значении переменной $\eta$ безразмерный потенциал

$$
V(x) \equiv \frac{2 m_{1} R^{2}}{\hbar^{2}} \widetilde{V}(r), \quad r=x|R|, \quad x=2 \rho|\eta|,
$$

подчиняется по переменной $\rho$ трем условиям:

$$
V(2 \rho|\eta|) \in \mathcal{C}_{(0, \infty)}^{0}, \quad \lim _{\rho \rightarrow 0} \rho^{2} V(2 \rho|\eta|)=0, \quad \lim _{\rho \rightarrow \infty} \rho^{2} V(2 \rho|\eta|)=0 .
$$

Всюду далее считается, что потенциал $V$ удовлетворяет ограничениям (2), переменная $\eta$ принимает любые конечные вещественные значения, кроме нулевого, а значения аргумента $q$ любые, но положительные.

Перечислим известные в теории двумерного рассеяния [7], [8], [10] соотношения.

Так как суммарный потенциал $V^{\mathrm{c}}(r)+\widetilde{V}(r)$ не зависит от угла $\varphi$, двумерная задача Шредингера для рассеяния частицы $p_{1}$ допускает разделение переменных $r$ и $\varphi$ и сводится к бесконечной, но счетной $(2 \lambda=-1,1,3, \ldots)$ совокупности радиальных краевых задач Шредингера. В кулоновских переменных $\rho$ и $\eta$ каждая из таких задач представляется в виде одномерного уравнения Шредингера

$$
\left[\partial_{\rho}^{2}-\frac{\lambda(\lambda+1)}{\rho^{2}}+1-\frac{2 \eta}{\rho}-4 \eta^{2} V(\sqrt{2 \rho|\eta|})\right] u_{\lambda}(\rho, \eta)=0, \quad \rho>0,
$$

с условием

$$
\lim _{\rho \rightarrow 0} \frac{u_{\lambda}(\rho, \eta)}{F_{\lambda}(\rho, \eta)}=N(\eta)
$$

и условием

$$
u_{\lambda}(\rho, \eta)=\sin \left[\rho-\eta \ln (2 \rho)-\pi \frac{\lambda}{2}+\delta_{\lambda}^{\mathrm{c}}(\eta)+\delta_{\lambda}(\eta)\right]+O\left(\rho^{-1}\right), \quad \rho \rightarrow \infty .
$$

Непрерывное в области $0 \leqslant \rho<\infty$ решение $u_{\lambda}$ задачи (3)-(5) считается регулярной волновой функцией рассеяния частицы $p_{1}$ в состоянии $|q, \lambda\rangle$ с квантовыми числами $q$ и $\lambda$.

Асимптотики (4) и (5) содержат регулярную функцию Кулона $F_{\lambda}$ и заранее неизвестные нормировочный множитель $N(\eta)$ и фазу $\delta_{\lambda}(\eta)$. Функции $\delta_{\lambda}^{\mathrm{c}}(\eta)$ и $\delta_{\lambda}(\eta)$ переменной $\eta$ имеют простой физический смысл. Сумма $\delta_{\lambda}^{\mathrm{c}}(\eta)+\delta_{\lambda}(\eta)$ есть полная фаза двумерного рассеяния частицы $p_{1}$ суперпозицией кулоновского потенциала $2 \eta / \rho$ и потенциала $V$ в состоянии $|q, \lambda\rangle$. Слагаемое $\delta_{\lambda}^{\mathrm{c}}(\eta)$ представляет собой фазу рассеяния кулоновским потенциалом, равную аргументу гамма-функции $\Gamma(\lambda+1+i \eta)$. Слагаемое $\delta_{\lambda}(\eta)$ есть искомая фаза рассеяния потенциалом $V$ в кулоновском поле. Эта фаза, вообще говоря, не равна фазе рассеяния $\tilde{\delta}_{\lambda}(q)$, порожденной потенциалом $V$ в отсутствие кулоновского взаимодействия. Равенство $\delta_{\lambda}=\tilde{\delta}_{\lambda}$ достигается в некулоновском пределе $|R| \rightarrow \infty$. В этом пределе параметр Зоммерфельда 
$\eta=1 / 2 k R$ и кулоновский потенциал $2 \eta / \rho, \rho=k r$, сходятся к нулю, а произведение $4 \eta^{2} V(2 \rho|\eta|)$ согласно равенству (1) вырождается в произведение функции $2 m_{1} /(k \hbar)^{2}$ от размерного волнового числа $k$ и размерного потенциала $\left.\widetilde{V}(r)\right|_{r=\rho / k}$.

Сечение $\sigma_{\lambda}^{\text {ca }}(\eta)$ рассеяния квантовой частицы $p_{1}$ в состоянии $|q, \lambda\rangle$ определяется формулой

$$
\sigma_{\lambda}^{\mathrm{ca}}(\eta)=8 R \eta \varepsilon_{\lambda}\left\{\sin \left[\delta_{\lambda}^{\mathrm{c}}(\eta)+\delta_{\lambda}(\eta)\right]\right\}^{2}, \quad \varepsilon_{\lambda}= \begin{cases}1, & 2 \lambda=-1 \\ 2, & 2 \lambda>-1\end{cases}
$$

Такое сечение в силу тождества

$$
\sin ^{2}\left(\delta_{\lambda}^{\mathrm{c}}+\delta_{\lambda}\right) \equiv \sin ^{2} \delta_{\lambda}^{\mathrm{c}}+\sin ^{2} \delta_{\lambda}+2 \sin \delta_{\lambda}^{\mathrm{c}} \sin \delta_{\lambda} \cos \left(\delta_{\lambda}^{\mathrm{c}}+\delta_{\lambda}\right)
$$

является суммой слагаемых

$$
\sigma_{\lambda}^{\mathrm{c}}(\eta) \equiv 8 R \eta \varepsilon_{\lambda} \sin ^{2} \delta_{\lambda}^{\mathrm{c}}(\eta), \quad \sigma_{\lambda}^{\mathrm{int}}(\eta) \equiv 16 R \eta \varepsilon_{\lambda} \sin \delta_{\lambda}^{\mathrm{c}} \sin \delta_{\lambda} \cos \left(\delta_{\lambda}^{\mathrm{c}}+\delta_{\lambda}\right)
$$

и слагаемого

$$
\sigma_{\lambda}(\eta) \equiv 8 R \eta \varepsilon_{\lambda} \sin ^{2} \delta_{\lambda}(\eta)=\frac{\sigma_{\lambda}^{\mathrm{u}}(\eta)}{\operatorname{ctg}^{2} \delta_{\lambda}(\eta)+1}, \quad \sigma_{\lambda}^{\mathrm{u}}(\eta) \equiv 8 R \eta \varepsilon_{\lambda} .
$$

Слагаемое $\sigma_{\lambda}^{\text {c }}$ есть сечение рассеяния одним кулоновским потенциалом. Слагаемое $\sigma_{\lambda}^{\text {int }}$ - вклад от интерференции рассеяния кулоновским потенциалом и потенциалом $V$. Слагаемое $\sigma_{\lambda}$ - сечение рассеяния этим потенциалом в кулоновском поле. При любом $\eta$ такое сечение не превышает своего унитарного предела $\sigma_{\lambda}^{\mathrm{u}}$.

В следующем разделе краевая задача Шредингера (3)-(5) будет исходной. Под ее полным решением подразумевается, во-первых, вычисление всех функций $N(\eta), u_{\lambda}$, $\delta_{\lambda}$ и $\sigma_{\lambda}$ при любых $\lambda$ и $\eta$, во-вторых, анализ поведения этих функций в трех физически интересных пределах $\lambda \rightarrow \infty, \eta \rightarrow 0$ и $q \rightarrow 0$. Случай $q \rightarrow 0$ будет исследован в следующей работе.

\section{3. ФОРМУЛИРОВКА МЕТОДА}

Напомним, что метод фазовых функций в теории трехмерного рассеяния [18] центральным потенциалом реализован в двух версиях: нелинейной [7] и линейной [19]. В первой из них ключевым является нелинейное дифференциальное уравнение первого порядка для фазовой функции, а во второй - система двух линейных дифференциальных уравнений первого порядка для амплитудных функций. В теории дифференциальных уравнений [20] эти функции называются "варьируемыми постоянными". Линейная и нелинейная версии метода фазовых функций успешно использовались ранее соответственно в работах [9], [10] и [11] для анализа двумерного рассеяния медленной квантовой частицы центральным короткодействующим потенциалом.

Предлагаемый подход к исчерпывающему решению радиальной задачи Шредингера (3)-(5) является линейной версией метода фазовых функций в теории двумерного рассеяния и для краткости называется методом амплитудных функций.

Формулировку предлагаемого метода амплитудных функций даем в три этапа; каждому из них посвятим отдельный пункт. В п. 3.1 выведена линейная задача 
Коши, наиболее удобная для численного анализа амплитудных функций. В п. 3.2 получены представления парциальных волновых функций, фаз и сечений двумерного рассеяния через амплитудные функции. В п. 3.3 задача Коши для амплитудных функций сведена к интегральным уравнениям и интегральным представлениям, наиболее адаптированным для доказательства теоремы существования и единственности таких функций.

3.1. Задача Коши для амплитудных функций. В случае потенциала $V$, тождественно равного нулю, уравнению (3) удовлетворяют линейно независимые вещественные волновые функции Кулона $F_{\lambda}(\rho, \eta)$ и $G_{\lambda}(\rho, \eta)$ полуцелого порядка $\lambda=-1 / 2,1 / 2, \ldots$. Эти функции детально исследованы в предыдущих работах [16], [17], а в настоящей работе используются в качестве вспомогательных.

Сведем радиальное уравнение Шредингера (3) с начальным условием (4) к задаче Коши для амплитудных функций $c(\rho, \eta)$ и $s(\rho, \eta)$, а именно к недиагональной системе двух линейных дифференциальных уравнений первого порядка:

$$
\begin{aligned}
& \partial_{\rho} c(\rho, \eta)=4 \eta^{2} V(2 \rho|\eta|)\left[c(\rho, \eta) F_{\lambda}(\rho, \eta)+s(\rho, \eta) G_{\lambda}(\rho, \eta)\right] G_{\lambda}(\rho, \eta), \\
& \partial_{\rho} s(\rho, \eta)=-4 \eta^{2} V(2 \rho|\eta|)\left[c(\rho, \eta) F_{\lambda}(\rho, \eta)+s(\rho, \eta) G_{\lambda}(\rho, \eta)\right] F_{\lambda}(\rho, \eta),
\end{aligned}
$$

с начальными условиями

$$
\left.c(\rho, \eta)\right|_{\rho=0}=1,\left.\quad s(\rho, \eta)\right|_{\rho=0}=0 .
$$

Применим известный в теории дифференциальных уравнений [20] метод вариации постоянных по той же схеме, что и в случае двумерного рассеяния квантовой частицы одним короткодействующим потенциалом [10].

Искомые функции $c$ и $s$ подчиним тождеству

$$
F_{\lambda}(\rho, \eta) \partial_{\rho} c(\rho, \eta)+G_{\lambda}(\rho, \eta) \partial_{\rho} s(\rho, \eta) \equiv 0, \quad \rho \geqslant 0
$$

Заметим, что из этого тождества следуют два представления:

$$
\partial_{\rho} s(\rho, \eta)=-\frac{F_{\lambda}(\rho, \eta)}{G_{\lambda}(\rho, \eta)} \partial_{\rho} c(\rho, \eta), \quad \partial_{\rho} c(\rho, \eta)=-\frac{G_{\lambda}(\rho, \eta)}{F_{\lambda}(\rho, \eta)} \partial_{\rho} s(\rho, \eta), \quad \rho \geqslant 0 .
$$

Решение $u_{\lambda}$ краевой задачи Шредингера (3)-(5) будем искать в виде произведения нормировочного множителя $N$ и вспомогательной функции $U$ :

$$
u_{\lambda}(\rho, \eta)=N(\eta) U(\rho, \eta), \quad U(\rho, \eta) \equiv c(\rho, \eta) F_{\lambda}(\rho, \eta)+s(\rho, \eta) G_{\lambda}(\rho, \eta)
$$

Сначала в уравнении (3) заменим функцию $u_{\lambda}$ произведением (11). Затем воспользуемся тем, что функции Кулона $F_{\lambda}$ и $G_{\lambda}$ удовлетворяют этому уравнению в случае выключенного потенциала $V$ и подчиняются тождеству (9). В результате получим уравнение

$$
\begin{aligned}
& \partial_{\rho} c(\rho, \eta) \partial_{\rho} F_{\lambda}(\rho, \eta)+\partial_{\rho} s(\rho, \eta) \partial_{\rho} G_{\lambda}(\rho, \eta)= \\
& \quad=4 \eta^{2} V(2 \rho|\eta|)\left[c(\rho, \eta) F_{\lambda}(\rho, \eta)+s(\rho, \eta) G_{\lambda}(\rho, \eta)\right]
\end{aligned}
$$


Исключим из этого уравнения производную $\partial_{\rho} s$. Для этого применим первое из двух равенств (10). Полученное уравнение упростим, используя соотношение Вронского [16]

$$
W\left(F_{\lambda}, G_{\lambda}\right) \equiv F_{\lambda}(\rho, \eta) \partial_{\rho} G_{\lambda}(\rho, \eta)-G_{\lambda}(\rho, \eta) \partial_{\rho} F_{\lambda}(\rho, \eta)=-1, \quad \rho \geqslant 0 .
$$

В результате выведем первое уравнение системы (7). Ее второе уравнение выведем аналогичным способом. Сначала, используя второе из равенств (10), сведем уравнение (12) к уравнению, не содержащему производную $\partial_{\rho} c$. Затем покажем, что благодаря соотношению Вронского полученное уравнение является вторым уравнением системы (7).

Теперь для этой системы выведем начальные условия (8). Используем краевое условие (4) для функции $u_{\lambda}$ и ее представления (11) через функции $N, U, c$ и $s$. Краевое условие (4) выполняется тогда и только тогда, когда

$$
U(\rho, \eta)=c(\rho, \eta) F_{\lambda}(\rho, \eta)+s(\rho, \eta) G_{\lambda}(\rho, \eta) \rightarrow F_{\lambda}(\rho, \eta), \quad \rho \rightarrow 0 .
$$

Функция $U$ сходится к функции $F_{\lambda}$ при $\rho \rightarrow 0$, если в этом пределе функция $c$ сходится к единице и выполняются следующие соотношения:

$$
s(\rho, \eta) G_{\lambda}(\rho, \eta)=o\left(c(\rho, \eta) F_{\lambda}(\rho, \eta)\right) \rightarrow 0, \quad \rho \rightarrow 0, \quad 2 \lambda \geqslant-1 .
$$

Эти соотношения в силу условия $c(\rho, \eta)=1$ при $\rho=0$ и известных асимптотик [16] при $\rho \rightarrow 0$,

$$
F_{\lambda}(\rho, \eta) \sim C_{\lambda}(\eta) \rho^{\lambda+1}, \quad G_{\lambda}(\rho, \eta) \sim \frac{1}{C_{\lambda}(\eta) \rho^{\lambda}} \begin{cases}-\ln \rho, & 2 \lambda=-1 \\ (2 \lambda+1)^{-1}, & 2 \lambda>-1\end{cases}
$$

означают, что

$$
s(\rho, \eta)=\left\{\begin{array}{ll}
o\left(\rho^{2 \lambda+1}\right), & 2 \lambda>-1, \\
o(1 / \ln \rho), & 2 \lambda=-1,
\end{array} \quad \rho \rightarrow 0 .\right.
$$

Следовательно, в пределе $\rho \rightarrow 0$ функция $s$ должна сходиться к нулю. Этим утверждением закончим вывод начальных условий (8).

Отметим, что указанные условия являются достаточными для того, чтобы волновая функция имела асимптотику (4), но основаны на двух предположениях: функции $c$ и $s$ непрерывны в точке $\rho=0$ справа, а функция $s$ обладает свойством (14). Эти предположения докажем в следующем разделе в теореме 4.

Перейдем к качественному анализу задачи Коши (7), (8).

Сначала обсудим функцию $U$. По определению (11) функция $U$ является суммой, первое слагаемое которой содержит функцию $F_{\lambda}$ с амплитудой $c$, а второе функцию $G_{\lambda}$ с амплитудой $s$. Поэтому функции $c$ и $s$ называются амплитудными. Если эти функции удовлетворяют задаче Коши (7), (8), то функция $U$ является решением уравнения Шредингера (3) с начальным условием $U / F_{\lambda} \rightarrow 1$ при $\rho \rightarrow 0$. Верно и обратное утверждение. Следовательно, уравнение Шредингера (3) с таким начальным условием эквивалентно задаче Коши (7), (8).

Заметим, что правые части уравнений (7) содержат произведения $V F_{\lambda}^{2}, V F_{\lambda} G_{\lambda}$ и $V G_{\lambda}^{2}$. Исследуем особенности поведения этих произведений в двух пределах $\rho \rightarrow \infty$ и $\rho \rightarrow 0$ и сформулируем следствия выявленных особенностей. 
Пусть $\rho \rightarrow \infty$. Согласно третьему из условий $(2)$ потенциал $V$ убывает, как $o\left(\rho^{-2}\right)$, а функции Кулона имеют осциллирующие асимптотики [16]:

$$
\begin{gathered}
F_{\lambda}(\rho, \eta) \sim \sin \chi_{\lambda}(\rho, \eta), \quad G_{\lambda}(\rho, \eta) \sim \cos \chi_{\lambda}(\rho, \eta), \\
\chi_{\lambda}(\rho, \eta) \equiv \rho-\eta \ln (2 \rho)-\pi \frac{\lambda}{2}+\delta_{\lambda}^{\mathrm{c}}(\eta) .
\end{gathered}
$$

Следовательно, при $\rho \rightarrow \infty$ и любом $\lambda$ произведения $V F_{\lambda}^{2}, V F_{\lambda} G_{\lambda}$ и $V G_{\lambda}^{2}$, осциллируя, сходятся к нулю, как $o\left(\rho^{-2}\right)$. Поэтому следует ожидать, что правые части уравнений (7), производные $\partial_{\rho} c, \partial_{\rho} s$ и функции $c$ и $s$ будут осциллировать в области $\rho \gg 1$.

Рассмотрим случай $\rho \rightarrow 0$ и $2 \lambda=-1$. Вследствие формул (13) при $\rho \rightarrow 0$ имеем три соотношения (напомним, $x=2 \rho|\eta|$ )

$$
\begin{gathered}
V(x) F_{\lambda}^{2}(\rho, \eta) \sim C_{\lambda}^{2}(\eta) \rho \cdot V(x), \quad V(x) F_{\lambda}(\rho, \eta) G_{\lambda}(\rho, \eta) \sim-\rho \ln \rho \cdot V(x), \\
V(x) G_{\lambda}^{2}(\rho, \eta) \sim C_{\lambda}^{-2}(\eta) \rho \ln ^{2} \rho \cdot V(x) .
\end{gathered}
$$

Первое из условий $(2)$ означает, что $V=o\left(\rho^{-2}\right)$ при $\rho \rightarrow 0$, и поэтому оно не является достаточным для того, чтобы правые части этих соотношений имели конечные пределы в точке $\rho=0$. Следовательно, произведения $V F_{\lambda}^{2}, V F_{\lambda} G_{\lambda}$ и $V G_{\lambda}^{2}$, вообще говоря, не являются непрерывными функциями в этой точке: их модули могут неограниченно возрастать при $\rho \rightarrow 0$.

Теперь пусть $\rho \rightarrow 0$, но $2 \lambda>-1$. Тогда $V=o\left(\rho^{-2}\right)$ и согласно формулам (13)

$$
\begin{gathered}
V(x) F_{\lambda}^{2}(\rho, \eta) \sim C_{\lambda}^{2}(\eta) \rho^{2 \lambda+1} V(x), \quad V(x) F_{\lambda}(\rho, \eta) G_{\lambda}(\rho, \eta) \sim \frac{\rho V(x)}{2 \lambda+1}, \\
V(x) G_{\lambda}^{2}(\rho, \eta) \sim \frac{V(x)}{\left[C_{\lambda}(\eta) \rho^{\lambda}(2 \lambda+1)\right]^{2}} .
\end{gathered}
$$

Следовательно, функция $V F_{\lambda}^{2}$ непрерывна в точке $\rho=0$ справа, но модули обеих функций $V F_{\lambda} G_{\lambda}$ и $V G_{\lambda}^{2}$ могут неограниченно возрастать, если $\rho \rightarrow 0$.

Итак, если $V=o\left(\rho^{-2}\right)$ при $\rho \rightarrow 0$, то функции $V F_{\lambda} G_{\lambda}$ и $V G_{\lambda}^{2}$ могут быть неограниченными в пределе $\rho \rightarrow 0$. Этому факту следует уделить особое внимание при численном интегрировании задачи Коши (7), (8) и при доказательстве существования и единственности ее решения $\{c, s\}$ на всей полуоси $\rho \geqslant 0$. Только в исключительном случае, когда все функции $V F_{\lambda}^{2}, V F_{\lambda} G_{\lambda}$ и $V G_{\lambda}^{2}$ непрерывны в точке $\rho=0$ справа, обсуждаемая задача Коши по теореме Пикаро [20] имеет на любом, но конечном отрезке $0 \leqslant \rho<b<\infty$ одно и только одно решение $\{c, s\}$. Однако и в этом случае из теоремы Пикаро не следует, что компоненты $c$ и $s$ такого решения ограничены в точке $\rho=\infty$ и принимают в этой точке вполне определенные значения.

Сформулируем основной результат настоящего пункта: дан вывод и качественный анализ задачи Коши (7), (8) для амплитудных функций $c$ и $s$. Как выяснилось, эта задача не адаптирована к простому доказательству теоремы существования и единственности функций $c$ и $s$. Чтобы доказать такую теорему в разделе 4 , придется в п. 3.3 вывести специальные интегральные уравнения и представления.

3.2. Представления нормировочного множителя, фазы и сечения рассеяния. Следует напомнить и пояснить принятое в математическом анализе определение непрерывности функции $g(\rho)$, заданной на полуоси $\rho \geqslant 0$. Функция $g(\rho)$ 
называется непрерывной на полуоси $\rho \geqslant 0$, если она непрерывна в каждой точке интервала $0<\rho<\infty$, непрерывна справа в точке $\rho=0$ и в пределе $\rho \rightarrow \infty$ принимает вполне определенное конечное значение. Функция, непрерывная на полуинтервале $0 \leqslant \rho<\infty$ и ограниченная в пределе $\rho \rightarrow \infty$, не всегда является непрерывной на полуоси $\rho \geqslant 0$. Например, функция $\sin \rho$ обладает такими свойствами, но не сходится ни к какому определенному значению при $\rho \rightarrow \infty$.

Предположим, что на всей полуоси $\rho \geqslant 0$ амплитудные функции $c$ и $s$ однозначно определяются задачей Коши (7), (8), являются непрерывными и не имеют общих нулей. Все эти предположения докажем в следующем разделе, а настоящий пункт посвятим выводу и обсуждению представлений функций $N, \delta_{\lambda}$ и $\sigma_{\lambda}$ через амплитудные функции $c$ и $s$. Во всех таких представлениях для сокращения записи символом $A(\eta)$ обозначаем предельное при $\rho \rightarrow \infty$ значение $A(\infty, \eta)$ исследуемой функции $A(\rho, \eta)$.

Сначала найдем асимптотику функции $u_{\lambda}$ в пределе $\rho \rightarrow \infty$. Используем представление этой функции в виде произведения (11). В этом произведении по предположению функции $c$ и $s$ всюду ограничены, а кулоновские функции имеют асимптотики (15). Поэтому искомая асимптотика функции $u_{\lambda}$ такова: при $\rho \rightarrow \infty$

$$
u_{\lambda}(\rho, \eta) \sim N(\eta) c(\rho, \eta) \sin \left[\chi_{\lambda}(\rho, \eta)+\delta_{\lambda}(\eta)\right]+N(\eta) s(\rho, \eta) \cos \left[\chi_{\lambda}(\rho, \eta)+\delta_{\lambda}(\eta)\right] .
$$

Теперь заметим, что полученная асимптотика совпадает с требуемой асимптотикой (5) тогда и только тогда, когда выполняются два предельных соотношения

$$
\lim _{\rho \rightarrow \infty} N(\eta) c(\rho, \eta)=\cos \delta_{\lambda}(\eta), \quad \lim _{\rho \rightarrow \infty} N(\eta) s(\rho, \eta)=\sin \delta_{\lambda}(\eta)
$$

Следовательно, нормировочный множитель $N(\eta)$ определяется формулами

$$
N(\eta)=\lim _{\rho \rightarrow \infty} N(\rho, \eta), \quad N(\rho, \eta) \equiv\left[c^{2}(\rho, \eta)+s^{2}(\rho, \eta)\right]^{-1 / 2},
$$

а тангенс фазы рассеяния $\delta_{\lambda}(\eta)$ - формулами

$$
\operatorname{tg} \delta_{\lambda}(\eta)=\lim _{\rho \rightarrow \infty} \operatorname{tg} \delta_{\lambda}(\rho, \eta), \quad \operatorname{tg} \delta_{\lambda}(\rho, \eta) \equiv \frac{s(\rho, \eta)}{c(\rho, \eta)} .
$$

Вследствие этих формул и определения (6) для сечения $\sigma_{\lambda}$ верно представление

$$
\sigma_{\lambda}(\eta)=\lim _{\rho \rightarrow \infty} \sigma_{\lambda}(\rho, \eta), \quad \sigma_{\lambda}(\rho, \eta)=\sigma_{\lambda}^{\mathrm{u}}(\eta)[s(\rho, \eta) N(\rho, \eta)]^{2}
$$

В силу высказанных выше предположений о свойствах амплитудных функций формулы (11) и (16)-(18) однозначно определяют обе функции $U$ и $u_{\lambda}$, нормировочный множитель $N(\eta)$, фазу и сечение рассеяния $\delta_{\lambda}(\eta)$ и $\sigma_{\lambda}(\eta)$. При тех же предположениях функции $u_{\lambda}(\rho, \eta), U(\rho, \eta)$ и функции $N(\rho, \eta), \delta_{\lambda}(\rho, \eta)$ и $\sigma_{\lambda}(\rho, \eta)$ являются непрерывными на всей полуоси $\rho \geqslant 0$. Если амплитудные функции $c$ и $s$ удовлетворяют задаче Коши $(7),(8)$, то функция $u_{\lambda}$, вычисленная по формулам (11) и (16), является решением исходной краевой задачи Шредингера (3)-(5). Верно и обратное утверждение. Следовательно, задача Шредингера (3)-(5) эквивалентна задаче Коши (7), (8), дополненной формулами (11) и (16)-(18). 
Важным следствием такой эквивалентности является следующее утверждение: если задача Коши (7), (8) имеет единственное решение $\{c, s\}$, компоненты которого $c$ и $s$ непрерывны на всей полуоси $\rho \geqslant 0$ и не имеют общих нулей, то краевая задача Шредингера (3)-(5) однозначно разрешима, а ее решение $u_{\lambda}$ непрерывно на полуинтервале $0 \leqslant \rho<\infty$.

Выявим физический смысл вспомогательных функций $N(\rho, \eta), \delta_{\lambda}(\rho, \eta)$ и $\sigma_{\lambda}(\rho, \eta)$. Для этого в системе (7) заменим потенциал $V(x)$ финитным потенциалом $V_{d}(x)$, совпадающим с потенциалом $V$ на некотором конечном отрезке $0 \leqslant x \leqslant d<\infty$ и равным нулю вне этого отрезка. В монографиях [7], [19] потенциал $V_{d}$ называется потенциалом $V$, “обрезанным" в точке $x=d$. Так как $x=2 \rho|\eta|$, такой точке отвечает точка $\rho=\rho_{d} \equiv d / 2|\eta|$. Предположим, что решение $\{c, s\}$ задачи Коши (7), (8) с "обрезанным" потенциалом $V$ найдено на всем отрезке $0 \leqslant \rho \leqslant \rho_{d}$. Построим непрерывное продолжение этого решения в область $\rho>\rho_{d}$, в которой "обрезанный" потенциал тождественно равен нулю. В этой области исследуемые уравнения (7) вырождаются в $\partial_{\rho} c=0$ и $\partial_{\rho} s=0$ с начальными условиями $c(\rho, \eta)=c\left(\rho_{d}, \eta\right)$ и $s(\rho, \eta)=s\left(\rho_{d}, \eta\right)$ в точке $\rho=\rho_{d}$. Такая задача Коши имеет единственное решение: $c(\rho, \eta)=c\left(\rho_{d}, \eta\right), s(\rho, \eta)=s\left(\rho_{d}, \eta\right)$ при любом $\rho \geqslant \rho_{d}$, в том числе и при $\rho=\infty$. Это решение и определения (16)-(18) порождают следующие соотношения:

$$
\begin{gathered}
N(\eta) \equiv \lim _{\rho \rightarrow \infty} N(\rho, \eta)=N\left(\rho_{d}, \eta\right) \\
\delta_{\lambda}(\eta) \equiv \lim _{\rho \rightarrow \infty} \delta_{\lambda}(\rho, \eta)=\delta_{\lambda}\left(\rho_{d}, \eta\right), \quad \sigma_{\lambda}(\eta) \equiv \lim _{\rho \rightarrow \infty} \sigma_{\lambda}(\rho, \eta)=\sigma_{\lambda}\left(\rho_{d}, \eta\right) .
\end{gathered}
$$

В силу этих соотношений обсуждаемые функции $N(\rho, \eta), \delta_{\lambda}(\rho, \eta)$ и $\sigma_{\lambda}(\rho, \eta)$ обладают прозрачным физическим смыслом: значения этих функций в любой точке $\rho=\rho_{d}$ являются нормировочным множителем $N(\eta)$, фазой $\delta_{\lambda}(\eta)$ и сечением $\sigma_{\lambda}(\eta)$ рассеяния в случае потенциала $V(x)$, “обрезанного" в точке $x=d=2 \rho_{d}|\eta|$. Благодаря такому смыслу функции $\delta_{\lambda}(\rho, \eta)$ ее можно назвать фазовой функцией.

3.3. Интегральные уравнения и интегральные представления. Дадим интегральную формулировку задач для функций $c, s$ и $U$.

Сначала представим исходную задачу Коши (7), (8) в виде эквивалентной ей системы двух интегральных уравнений Вольтерра второго рода:

$$
\begin{aligned}
& c(\rho, \eta)=1+4 \eta^{2} \int_{0}^{\rho} V(2 t|\eta|) U(t, \eta) G_{\lambda}(t, \eta) d t \\
& s(\rho, \eta)=-4 \eta^{2} \int_{0}^{\rho} V(2 t|\eta|) U(t, \eta) F_{\lambda}(t, \eta) d t .
\end{aligned}
$$

В этих уравнения для краткости записи использовалась функция $U$. Напомним, что функция $U$ определяется через амплитудные функции $c$ и $s$ формулами (11), удовлетворяет уравнению Шредингера (3) с начальным условием $U / F_{\lambda} \rightarrow 1$ при $\rho \rightarrow 0$ и связана с радиальной волновой функцией $u_{\lambda}$ равенством $U=u_{\lambda} / N$, где множитель $N$ обеспечивает нормировку (5).

Выведем интегральное уравнение для функции $U$. Для этого в ее представлении (11) через амплитудные функции $c$ и $s$ заменим последние правыми частями 
соотношений (19) и в результате получим искомое интегральное уравнение Вольтерра второго рода

$$
\begin{aligned}
U(\rho, \eta)= & F_{\lambda}(\rho, \eta)+ \\
& +4 \eta^{2} \int_{0}^{\rho}\left[F_{\lambda}(\rho, \eta) G_{\lambda}(t, \eta)-G_{\lambda}(\rho, \eta) F_{\lambda}(t, \eta)\right] V(2 t|\eta|) U(t, \eta) d t .
\end{aligned}
$$

В теории трехмерного рассеяния [18] уравнением Липпмана-Швингера принято называть интегральное уравнение, эквивалентное радиальному уравнению Шредингера с начальным условием в точке $\rho=0$. Поэтому и в теории двумерного рассеяния вполне логичным представляется называть уравнение (20) уравнением Липпмана-Швингера.

Отметим, что предложенная редукция уравнения Шредингера (3) к уравнению Липпмана-Швингера (20), основанная на использовании функций $c$ и $s$, является новым способом, отличным от давно известного в теории дифференциальных уравнений [20].

Обсудим уравнения (19) и (20). В уравнениях (19) символом $U$ обозначена сумма произведений $c F_{\lambda}$ и $s G_{\lambda}$. Следовательно, правые части уравнений (19) содержат обе искомые функции $c$ и $s$. Значит, система этих уравнений не диагональная, и уже поэтому ее сложно исследовать методом последовательных приближений Пикара-Линделефа [20]. Применение этого метода к уравнениям (19) и (20) представляется невозможным из-за отсутствия мажорантных оценок произведения функций $F_{\lambda}\left(\rho^{\prime}, \eta\right)$ и $G_{\lambda}(\rho, \eta)$ при условии $\rho<\rho^{\prime}$. Если функция $U$ найдена, например, как решение уравнения (20), то соотношения (19) становятся интегральными представлениями функций $c$ и $s$ через функции $F_{\lambda}, G_{\lambda}$ и $U$.

Перейдем к выводу еще одной системы интегральных уравнений, а именно, покажем, что задачу Коши (7), (8) для амплитудных функций $c$ и $s$ можно свести к диагональной системе двух интегральных уравнений

$$
\begin{aligned}
& y_{1}(\rho, \eta)=1+\int_{0}^{\rho} P_{1}\left(\rho_{1}, \eta\right) d \rho_{1} \int_{0}^{\rho_{1}} P_{2}\left(\rho_{2}, \eta\right) y_{1}\left(\rho_{2}, \eta\right) d \rho_{2}, \\
& y_{2}(\rho, \eta)=\int_{0}^{\rho} P_{2}\left(\rho_{1}, \eta\right) d \rho_{1}+\int_{0}^{\rho} P_{2}\left(\rho_{1}, \eta\right) d \rho_{1} \int_{0}^{\rho_{1}} P_{1}\left(\rho_{2}, \eta\right) y_{2}\left(\rho_{2}, \eta\right) d \rho_{2},
\end{aligned}
$$

в которых $\rho \geqslant 0$, а функции

$$
\begin{aligned}
& P_{1}(\rho, \eta) \equiv 4 \eta^{2} V(2 \rho|\eta|) G_{\lambda}^{2}(\rho, \eta) e^{2 B(\rho, \eta)} \\
& P_{2}(\rho, \eta) \equiv-4 \eta^{2} V(2 \rho|\eta|) F_{\lambda}^{2}(\rho, \eta) e^{-2 B(\rho, \eta)}
\end{aligned}
$$

содержат интеграл

$$
B(\rho, \eta) \equiv 4 \eta^{2} \int_{0}^{\rho} V(2 t|\eta|) F_{\lambda}(t, \eta) G_{\lambda}(t, \eta) d t .
$$

Положив в исследуемой задаче (7), (8)

$$
c(\rho, \eta)=y_{1}(\rho, \eta) e^{B(\rho, \eta)}, \quad s(\rho, \eta)=y_{2}(\rho, \eta) e^{-B(\rho, \eta)},
$$

выведем недиагональную систему двух дифференциальных уравнений

$$
\partial_{\rho} y_{1}(\rho, \eta)=P_{1}(\rho, \eta) y_{2}(\rho, \eta), \quad \partial_{\rho} y_{2}(\rho, \eta)=P_{2}(\rho, \eta) y_{1}(\rho, \eta), \quad \rho>0,
$$


с начальными условиями $y_{1}=1$ и $y_{2}=0$ в точке $\rho=0$. Запишем такую задачу Коши в виде системы двух интегральных уравнений

$$
y_{1}(\rho, \eta)=1+\int_{0}^{\rho} P_{1}(t, \eta) y_{2}(t, \eta) d t, \quad y_{2}(\rho, \eta)=\int_{0}^{\rho} P_{2}(t, \eta) y_{1}(t, \eta) d t, \quad \rho \geqslant 0 .
$$

Затем в первом из этих уравнений заменим функцию $y_{2}$ правой частью второго уравнения, а во втором уравнении заменим функцию $y_{1}$ правой частью первого уравнения. В результате такой итерации получится система двух уравнений (21), которую и хотелось вывести.

Обсудим уравнения (21) и (25). Первое из уравнений системы (21) не содержит функцию $y_{2}$, а второе не содержит функцию $y_{1}$. Поэтому оба обсуждаемых уравнения можно исследовать независимо друг от друга. В этом заключается первое и неоспоримое преимущество системы (21) по сравнению с недиагональной системой (19). Второе преимущество состоит в том, что достаточно решить только первое из уравнений системы (21), а затем вычислить функцию $y_{2}$ как интеграл (25), содержащий уже найденную функцию $y_{1}$.

Итак, уравнения (7) для функций $c$ и $s$ с начальными условиями (8) в точке $\rho=0$ весьма просто сводятся к интегральным уравнениям (21). Обобщим этот способ на случай начальных условий, заданных в произвольной точке $b$ полуоси $\rho>0$.

Сначала сформулируем новую задачу Коши для функций $c$ и $s$. Пусть $z_{1}(b, \eta)$ и $z_{2}(b, \eta)$ - известные функции переменной $\eta$. Для уравнений (7) используем начальные условия

$$
\left.c(\rho, \eta)\right|_{\rho=b}=z_{1}(b, \eta),\left.\quad s(\rho, \eta)\right|_{\rho=b}=z_{2}(b, \eta), \quad b>0 .
$$

Теперь покажем, что новая задача Коши (7), (26) на всей полуоси $\rho \geqslant 0$ сводится к диагональной системе двух интегральных уравнений

$$
z_{i}(\rho, \eta)=z_{i 0}(\rho, \eta)+\int_{b}^{\rho} T_{i}\left(\rho_{1}, \eta\right) d \rho_{1} \int_{b}^{\rho_{1}} T_{j}\left(\rho_{2}, \eta\right) z_{i}\left(\rho_{2}, \eta\right) d \rho_{2}, \quad i=1,2 .
$$

Здесь и далее $j=2$, если $i=1$, и $j=1$, если $i=2$; функции $z_{i 0}$ задаются как

$$
z_{i 0}(\rho, \eta) \equiv z_{i}(b, \eta)+z_{j}(b, \eta) \int_{b}^{\rho} T_{i}(t, \eta) d t, \quad i=1,2,
$$

а функции

$$
\begin{aligned}
& T_{1}(\rho, \eta) \equiv 4 \eta^{2} V(2 \rho|\eta|) G_{\lambda}^{2}(\rho, \eta) e^{2 D(\rho, \eta)} \\
& T_{2}(\rho, \eta) \equiv-4 \eta^{2} V(2 \rho|\eta|) F_{\lambda}^{2}(\rho, \eta) e^{-2 D(\rho, \eta)}
\end{aligned}
$$

содержат интеграл

$$
D(\rho, \eta) \equiv 4 \eta^{2} \int_{b}^{\rho} V(2 t|\eta|) F_{\lambda}(t, \eta) G_{\lambda}(t, \eta) d t
$$

Положив в исследуемой задаче (7), (26)

$$
c(\rho, \eta)=z_{1}(\rho, \eta) e^{D(\rho, \eta)}, \quad s(\rho, \eta)=z_{2}(\rho, \eta) e^{-D(\rho, \eta)},
$$


выведем недиагональную систему двух дифференциальных уравнений

$$
\partial_{\rho} z_{i}(\rho, \eta)=T_{i}(\rho, \eta) z_{j}(\rho, \eta), \quad i=1,2,
$$

с начальными условиями $z_{i}(\rho, \eta)=z_{i}(b, \eta), i=1,2$, в точке $\rho=b$. Представим такую задачу Коши в виде системы двух интегральных уравнений

$$
z_{i}(\rho, \eta)=z_{i}(b, \eta)+\int_{b}^{\rho} T_{i}(t, \eta) z_{j}(t, \eta) d t, \quad i=1,2 .
$$

С помощью итерации сведем эти уравнения к уравнениям (27), которые и требовалось получить.

Поясним основные свойства уравнений (27), (32) и оптимальный способ их решения. Нетрудно проверить, что в частном случае $b=0$ эти уравнения вырождаются в уравнения (21) и (25). Оба уравнения (27) можно исследовать независимо друг от друга. Достаточно решить только первое из этих уравнений, а затем вычислить функцию $z_{2}$ как интеграл (32), содержащий уже найденную функцию $z_{1}$. При такой последовательности вычисления функций $z_{1}$ и $z_{2}$ функция $z_{1}$ является оригиналом, а функция $z_{2}-$ интегральным образом функции $z_{1}$.

\section{4. МАТЕМАТИЧЕСКОЕ ОБОСНОВАНИЕ МЕТОДА}

Дадим полное математическое обоснование метода амплитудных функций по следующему плану. В п. 4.1 доказаны три леммы о мажорантных оценках. В п. 4.2 эти леммы использованы для доказательства теорем существования и единственности решений систем интегральных уравнений (21), (27) и задачи Коши (7), (8).

4.1. Леммы о мажорантных оценках. Настоящий пункт содержит доказательства трех вспомогательных утверждений, а именно лемм 1-3 о мажорантных оценках функций (22) и (29). Такие оценки потребуются в следующем пункте для исследования систем интегральных уравнений (21) и (27). Сформулированные в леммах 1-3 утверждения представляют собой следствия единственных известных к настоящему времени мажорантных оценок [17] функций Кулона полуцелого порядка. В случае $2 \lambda>-1$ такие оценки настолько просты, что позволяют доказать лемму 1 без особых затруднений при любом $\rho \geqslant 0$. Леммы 2 и 3 посвящены особому случаю $2 \lambda=-1$. В этом случае для функций Кулона имеются три мажорантные оценки: $Q$, $Q_{-}$и $Q_{+}$. Оценка $Q$, наиболее точная из них, верна при любом $\rho \geqslant 0$, но содержит эллиптический интеграл первого рода. Все попытки использовать такую сложную оценку не увенчались успехом. Менее точные, зато простые, но функционально разные оценки $Q_{-}$и $Q_{+}$справедливы соответственно на отрезке $0 \leqslant \rho \leqslant b=0.05$ и полуоси $\rho \geqslant b$. Вследствие отмеченных выше свойств оценок $Q, Q_{-}$и $Q_{+}$анализ случая $2 \lambda=-1$ пришлось разбить на две леммы: в лемме 2 используется оценка $Q_{-}$, а в лемме 3 - оценка $Q_{+}$. Такое разбиение оказалось удачным: благодаря ему удалось найти наиболее простые и однотипные доказательства лемм 1-3. В лемме 1 все промежуточные выкладки снабжаются подробными пояснениями, что позволяет в леммах 2 и 3 перечислить лишь ключевые моменты их доказательств. 
ЛЕмма 1. Пусть $2 \lambda>-1$ и по определению

$$
\begin{gathered}
L(\rho, \eta) \equiv 4 a_{\lambda} \eta^{2} \int_{0}^{\rho} t|V(2 t|\eta|)| d t \\
a_{\lambda} \equiv \sqrt{\frac{2 \pi}{2 \lambda+1}}, \quad 2 \tau(\rho, \eta) \equiv e^{2 L(\rho, \eta)}-1 .
\end{gathered}
$$

Тогда произведение $P_{1} P_{2}$ бункиий (22) удовлетворяет неравенству

$$
\left|P_{1}(\rho, \eta) P_{2}\left(\rho^{\prime}, \eta\right)\right| \leqslant \partial_{\rho} \tau(\rho, \eta) \partial_{\rho^{\prime}} \tau\left(\rho^{\prime}, \eta\right), \quad 0 \leqslant \rho^{\prime} \leqslant \rho \leqslant \infty,
$$

и имеет место оценка

$$
\left|P_{2}(\rho, \eta)\right| \leqslant \max _{0 \leqslant t \leqslant \rho}\left|F_{\lambda}(t, \eta)\right| \partial_{\rho} \tau(\rho, \eta), \quad 0 \leqslant \rho \leqslant \infty .
$$

ДоКАЗАТЕЛЬСТво начнем с анализа интеграла $L$ и функции $\tau$.

Напомним, что $|\eta|=1 / 2 q, \rho=q x$ и поэтому $x=2 \rho|\eta|=r /|R|$. В интеграле $L$ перейдем к переменной интегрирования $x_{1}=2 t|\eta|=t / q$, верхний предел $\rho$ выразим через переменную $x=\rho / q$ и в итоге получим

$$
L(\rho, \eta)=\sqrt{\frac{2 \pi}{2 \lambda+1}} \int_{0}^{x} x_{1}\left|V\left(x_{1}\right)\right| d x_{1}, \quad x=\frac{\rho}{q}=2 \rho|\eta| .
$$

Следовательно, интеграл $L$ является функцией переменной $x=2 \rho|\eta|$ и поэтому не зависит от волнового числа $q$. Так как потенциал $V$ удовлетворяет условиям (2), интеграл $L$ сходится на нижнем пределе и существует при любом $\rho>0$, в том числе при $\rho=\infty$. В пределе $\lambda \rightarrow \infty$ интеграл $L$ убывает, как $O\left(\lambda^{-1 / 2}\right)$, при любых $\rho>0$ и $\eta \in(-\infty, \infty)$.

Теперь укажем важные свойства функции $\tau$, вытекающие из ее определения (33) через интеграл $L$ и из перечисленных выше свойств этого интеграла. Функция $\tau$ равна нулю в точке $\rho=0$, монотонно возрастает, но ограничена на полуоси $\rho \geqslant 0$ и убывает в пределе $\lambda \rightarrow \infty$, как $O\left(\lambda^{-1 / 2}\right)$. При этом производная $\partial_{\rho} \tau$ ограничена в области $\rho>0$, вообще говоря, неограничена в точке $\rho=0$, но интегрируема на всей полуоси $\rho \geqslant 0$.

Перейдем к выводу вспомогательных неравенств. Сначала покажем, что модуль интеграла $B$, заданного формулой $(23)$, не превосходит интеграла $L$. Затем перечислим важные следствия этого свойства.

Ясно, что модуль интеграла $B$ меньше интеграла с теми же пределами, но от модуля той же подынтегральной функции. Такой модуль не превосходит произведения модуля потенциала $V$ на правую часть известной мажорантной оценки [17]

$$
\left|F_{\lambda}\left(\rho^{\prime}, \eta\right) G_{\lambda}(\rho, \eta)\right| \leqslant a_{\lambda} \sqrt{\rho^{\prime} \rho}, \quad 0 \leqslant \rho^{\prime} \leqslant \rho \leqslant \infty, \quad 2 \lambda>-1 .
$$

Таким образом, верна цепочка следующих друг из друга неравенств

$$
\begin{aligned}
|B(\rho, \eta)| & =4 \eta^{2}\left|\int_{0}^{\rho} V(2 t|\eta|) F_{\lambda}(t, \eta) G_{\lambda}(t, \eta) d t\right| \leqslant \\
& \leqslant 4 \eta^{2} \int_{0}^{\rho}|V(2 t|\eta|)|\left|F_{\lambda}(t, \eta) G_{\lambda}(t, \eta)\right| d t \leqslant \\
& \leqslant 4 a_{\lambda} \eta^{2} \int_{0}^{\rho} t|V(2 t|\eta|)| d t=L(\rho, \eta)<\infty, \quad \rho \geqslant 0 .
\end{aligned}
$$


В силу этой цепочки интеграл $L(\rho, \eta)$ служит мажорантой интеграла $B(\rho, \eta)$ при любом $\rho \geqslant 0$. Поэтому всегда верны неравенства

$$
e^{ \pm 2 B(\rho, \eta)} \leqslant e^{2 L(\rho, \eta)}<\infty, \quad \rho \geqslant 0 .
$$

Вследствие этих неравенств и определений (33) при любом $\rho \geqslant 0$ имеем

$$
\begin{aligned}
4 a_{\lambda} \eta^{2} \rho|V(2 \rho|\eta|)| e^{ \pm 2 B(\rho, \eta)} & \leqslant 4 a_{\lambda} \eta^{2} \rho|V(2 \rho|\eta|)| e^{2 L(\rho, \eta)}= \\
& =\partial_{\rho} L(\rho, \eta) e^{2 L(\rho, \eta)}=\partial_{\rho} \tau(\rho, \eta) .
\end{aligned}
$$

Теперь докажем неравенство (34). Используя определение (22) функций $P_{1}$ и $P_{2}$, оценку (36) и соотношения (37), при условии $0 \leqslant \rho^{\prime} \leqslant \rho \leqslant \infty$ получаем

$$
\begin{aligned}
& \left|P_{1}(\rho, \eta) P_{2}\left(\rho^{\prime}, \eta\right)\right| \leqslant \\
& \quad \leqslant\left\{4 \eta^{2}|V(2 \rho|\eta|)| e^{2 B(\rho, \eta)}\right\}\left\{4 \eta^{2}\left|V\left(2 \rho^{\prime}|\eta|\right)\right| e^{-2 B\left(\rho^{\prime}, \eta\right)}\right\}\left|G_{\lambda}(\rho, \eta) F_{\lambda}\left(\rho^{\prime}, \eta\right)\right|^{2} \leqslant \\
& \quad \leqslant\left\{4 a_{\lambda} \eta^{2} \rho|V(2 \rho|\eta|)| e^{2 L(\rho, \eta)}\right\}\left\{4 a_{\lambda} \eta^{2} \rho^{\prime}\left|V\left(2 \rho^{\prime}|\eta|\right)\right| e^{2 L\left(\rho^{\prime}, \eta\right)}\right\}= \\
& \quad=\partial_{\rho} \tau(\rho, \eta) \partial_{\rho^{\prime}} \tau\left(\rho^{\prime}, \eta\right) .
\end{aligned}
$$

Следовательно неравенство (34) верно.

Осталось оценить функцию $P_{2}$. По определению $(22)$ эта функция содержит произведение двух функций $F_{\lambda}$. Модуль одной из них заменим максимальным значением функции $\left|F_{\lambda}(t, \eta)\right|$ на отрезке $0 \leqslant t \leqslant \rho$, а модуль другой - правой частью известного неравенства [17]

$$
\left|F_{\lambda}(\rho, \eta)\right| \leqslant a_{\lambda} \rho, \quad \rho \geqslant 0, \quad 2 \lambda>-1,
$$

затем воспользуемся соотношениями (37) и в итоге получим оценку (35). Приведем для большей ясности ее подробный вывод,

$$
\begin{aligned}
\left|P_{2}(\rho, \eta)\right| & \leqslant 4 \eta^{2}|V(2 \rho|\eta|)| F_{\lambda}^{2}(\rho, \eta) e^{-2 B(\rho, \eta)} \leqslant \\
& \leqslant \max _{0 \leqslant t \leqslant \rho}\left|F_{\lambda}(t, \eta)\right| 4 a_{\lambda} \eta^{2} \rho|V(2 \rho|\eta|)| e^{-2 B(\rho, \eta)} \leqslant \\
& \leqslant \max _{0 \leqslant t \leqslant \rho}\left|F_{\lambda}(t, \eta)\right| \partial_{\rho} L(\rho) e^{2 L(\rho)}=\max _{0 \leqslant t \leqslant \rho}\left|F_{\lambda}(t, \eta)\right| \partial_{\rho} \tau(\rho),
\end{aligned}
$$

и тем самым завершим доказательство леммы.

Лемма 2. Пусть $2 \lambda=-1, b=0.05, e-$ основание натурального логарифма, $B$ интеграл $(23), 0 \leqslant \rho \leqslant b$ и по определению

$$
\begin{aligned}
& L_{1}(\rho, \eta) \equiv 2 \eta^{2} \int_{0}^{\rho} t \ln ^{2}\left(\beta_{1} t^{2}\right)|V(2 t|\eta|)| d t \\
& 2 \tau_{1}(\rho, \eta) \equiv e^{2 L_{1}(\rho, \eta)}-1, \quad \beta_{1} \equiv \frac{1}{e} \sqrt{\frac{\pi}{2}}
\end{aligned}
$$

Тогда функиии (22) удовлетворяют неравенствам

$$
\begin{array}{cl}
\left|P_{1}(\rho, \eta) P_{2}\left(\rho^{\prime}, \eta\right)\right| \leqslant \partial_{\rho} \tau_{1}(\rho, \eta) \partial_{\rho^{\prime}} \tau_{1}\left(\rho^{\prime}, \eta\right), & 0 \leqslant \rho^{\prime} \leqslant \rho \leqslant b, \\
\left|P_{2}(\rho, \eta)\right| \leqslant \rho \partial_{\rho} \tau_{1}(\rho, \eta), & 0 \leqslant \rho \leqslant b .
\end{array}
$$


ДокАЗАТЕЛЬСтво начнем с анализа интеграла $L_{1}$ и функции $\tau_{1}$.

Интеграл $L_{1}$ зависит от волнового числа $q$ и существует, потому что согласно ограничениям (2) потенциал $V$ непрерывен на полуинтервале $0<\rho \leqslant b$ и удовлетворяет условию $V(2 t|\eta|)=o\left((t|\eta|)^{-2}\right)$ при $t \rightarrow+0$. Функция $\tau_{1}$ равна нулю в точке $\rho=0$, монотонно возрастает, но ограничена на всем отрезке $0 \leqslant \rho \leqslant b$. Производная $\partial_{\rho} \tau_{1}$ ограничена на полуинтервале $0<\rho \leqslant b$ и интегрируема на отрезке $[0, \rho]$, где $\rho \leqslant b$.

Теперь из известных оценок [17] функций Кулона

$$
\begin{aligned}
\left|F_{\lambda}\left(\rho^{\prime}, \eta\right) G_{\lambda}(\rho, \eta)\right| & \leqslant-\frac{\sqrt{\rho^{\prime} \rho}}{2} \ln \left(\beta_{1} \rho^{\prime} \rho\right), & & 0 \leqslant \rho^{\prime} \leqslant \rho \leqslant b, \\
\left|F_{\lambda}(\rho, \eta)\right| & \leqslant-\frac{\rho}{2} \ln \left(\beta_{1} \rho^{2}\right), & & 0 \leqslant \rho \leqslant b,
\end{aligned}
$$

где $\beta_{1}=(1 / e) \sqrt{\pi / 2}=0.4610685 \ldots$, выведем специальные оценки, позволяющие использовать доказательство леммы 1.

В частном случае $\rho=\rho^{\prime}$ из соотношений (40) следуют две мажорантные оценки

$$
F_{\lambda}^{2}(\rho, \eta) \leqslant \frac{\rho^{2}}{4} \ln ^{2}\left(\beta_{1} \rho^{2}\right), \quad\left|F_{\lambda}(\rho, \eta) G_{\lambda}(\rho, \eta)\right| \leqslant \frac{\rho}{2}\left|\ln \left(\beta_{1} \rho^{2}\right)\right|, \quad 0 \leqslant \rho \leqslant b .
$$

Вторая из них порождает менее точную оценку

$$
\left|F_{\lambda}(\rho, \eta) G_{\lambda}(\rho, \eta)\right| \leqslant \frac{\rho}{2} \ln ^{2}\left(\beta_{1} \rho^{2}\right), \quad 0 \leqslant \rho \leqslant b .
$$

Действительно, на отрезке $0 \leqslant \rho \leqslant 0.05$ все значения функции $\left|\ln \left(\beta_{1} \rho^{2}\right)\right|$ не превосходят числа $\left|\ln \left(0.05 \beta_{1}\right)\right|=6.765673 \ldots$, которое больше единицы. Следовательно, на этом отрезке функция $\left|\ln \left(\beta_{1} \rho^{2}\right)\right|$ всегда меньше своего квадрата.

В случае $\rho^{\prime}=\rho_{2}$ и $\rho=\rho_{1}$ благодаря соотношениям (40) верно неравенство

$$
\left|G_{\lambda}^{2}\left(\rho_{1}, \eta\right) F_{\lambda}^{2}\left(\rho_{2}, \eta\right)\right| \leqslant \frac{1}{4} \rho_{1} \rho_{2} \ln ^{2}\left(\beta_{1} \rho_{1} \rho_{2}\right), \quad 0 \leqslant \rho_{2} \leqslant \rho_{1} \leqslant b .
$$

Из этого неравенства в силу формул

$$
\ln ^{2}\left(\beta_{1} \rho_{1}^{2}\right)>1, \quad\left|\ln \left(\beta_{1} \rho_{1} \rho_{2}\right)\right| \leqslant\left|\ln \left(\beta_{1} \rho_{2}^{2}\right)\right|, \quad 0 \leqslant \rho_{2} \leqslant \rho_{1} \leqslant b,
$$

следует менее точная оценка

$$
G_{\lambda}^{2}\left(\rho_{1}, \eta\right) F_{\lambda}^{2}\left(\rho_{2}, \eta\right) \leqslant\left\{\frac{\rho_{1}}{2} \ln ^{2}\left(\beta_{1} \rho_{1}^{2}\right)\right\}\left\{\frac{\rho_{2}}{2} \ln ^{2}\left(\beta_{1} \rho_{2}^{2}\right)\right\}, \quad 0 \leqslant \rho_{2} \leqslant \rho_{1} \leqslant b,
$$

в которой первый или второй из сомножителей, заключенных в фигурные скобки, зависит только от одной переменной $\rho_{1}$ или $\rho_{2}$.

Теперь несложно доказать неравенства (39) по аналогии с доказательством неравенств (34), подробно изложенным в лемме 1. Для этого вместо оценок (36) и (38) функций Кулона используем (41)-(43), а вместо функций $L$ и $\tau-$ функции $L_{1}$ и $\tau_{1}$.

В силу определений интегралов $B$ и $L_{1}$ и оценки (42) верны два неравенства

$$
|B(\rho, \eta)| \leqslant L_{1}(\rho, \eta), \quad e^{ \pm 2 B(\rho, \eta)} \leqslant e^{2 L_{1}(\rho, \eta)}, \quad 0 \leqslant \rho \leqslant b .
$$


Благодаря второму из них, а также определениям функций $P_{1}, P_{2}, \tau_{1}$ и оценке (43) справедливо первое из неравенств (39). Второе из них есть следствие оценки (41) для функции $F_{\lambda}^{2}$. Ключевыми при выводе обоих неравенств (39) являются два тождества

$$
\partial_{\rho} \tau_{1}(\rho, \eta) \equiv \partial_{\rho} L_{1}(\rho, \eta) e^{2 L_{1}(\rho, \eta)} \equiv 2 \eta^{2} \rho \ln ^{2}\left(\beta_{1} \rho^{2}\right)|V(2 \rho|\eta|)| e^{2 L_{1}(\rho, \eta)} .
$$

Этим замечанием закончим доказательство леммы.

Лемма 3. Пусть $2 \lambda=-1, b=0.05, K(\pi / 4)$ - полный эллиптический интеграл первого рода, $D$ - интеграл (30), $\rho \geqslant b$ и по определению

$$
\begin{gathered}
L_{2}(\rho, \eta) \equiv 4 \beta_{2} \eta^{2} \int_{b}^{\rho} \sqrt{t}|V(2 t|\eta|)| d t \\
2 \tau_{2}(\rho, \eta) \equiv e^{2 L_{2}(\rho, \eta)}-1, \quad \beta_{2} \equiv \frac{2}{\sqrt{\pi}} K\left(\frac{\pi}{4}\right) .
\end{gathered}
$$

Тогда функции (29) удовлетворяют неравенствам

$$
\begin{aligned}
\left|T_{1}(\rho, \eta) T_{2}\left(\rho^{\prime}, \eta\right)\right| & \leqslant \partial_{\rho} \tau_{2}(\rho, \eta) \partial_{\rho^{\prime}} \tau_{2}\left(\rho^{\prime}, \eta\right), & & b \leqslant \rho^{\prime} \leqslant \rho \leqslant \infty \\
\left|T_{2}(\rho, \eta)\right| & \leqslant \max _{b \leqslant t \leqslant \rho}\left|F_{\lambda}(t, \eta)\right| \partial_{\rho} \tau_{2}(\rho, \eta), & & \rho \geqslant b,
\end{aligned}
$$

и при любом $\rho \geqslant b$ выполняются соотношения

$$
\left|\int_{b}^{\rho} T_{1}(t, \eta) d t\right| \leqslant p(\eta) \equiv 4 \eta^{2} \int_{b}^{\infty} G_{\lambda}^{2}(t, \eta)|V(2 t|\eta|)| e^{2 D(t, \eta)} d t<\infty .
$$

ДокАЗАТЕЛЬСтво начнем с обсуждения интеграла $L_{2}$ и функции $\tau_{2}$.

Интеграл $L_{2}$ зависит от волнового числа $q$ и существует при любом $\rho \geqslant b$ благодаря ограничениям (2) на потенциал $V$. В случае $\rho=\infty$ сходимость этого интеграла на верхнем пределе обеспечивается тем, что $V(2 t|\eta|)=o\left((t|\eta|)^{-2}\right)$ при $t \rightarrow \infty$. Функция $\tau_{2}$ равна нулю в точке $\rho=b$, монотонно возрастает на полуоси $\rho \geqslant b$, но ограничена в точке $\rho=\infty$.

Продолжение доказательства отличается от доказательства леммы 1 только тем, что вместо функций $B, L$ и $\tau$ используются функции $D, L_{2}$ и $\tau_{2}$, а вместо оценок $(36)$ и (38) - оценки [17]

$$
\begin{aligned}
\left|F_{\lambda}\left(\rho^{\prime}, \eta\right) G_{\lambda}(\rho, \eta)\right| & \leqslant \beta_{2}\left(\rho^{\prime} \rho\right)^{1 / 4} \\
\left|F_{\lambda}(\rho, \eta)\right| & \leqslant \beta_{2} \sqrt{\rho}
\end{aligned}
$$

Первая из этих оценок порождает неравенства

$$
|D(\rho, \eta)| \leqslant L_{2}(\rho, \eta), \quad e^{ \pm 2 D(\rho, \eta)} \leqslant e^{2 L_{2}(\rho, \eta)}, \quad \rho \geqslant b .
$$

Используя эти неравенства, определения (29) функций $T_{1}$ и $T_{2}$, оценку (46) произведения $F_{\lambda} G_{\lambda}$ и тождества

$$
\partial_{\rho} \tau_{2}(\rho, \eta) \equiv \partial_{\rho} L_{2}(\rho, \eta) e^{2 L_{2}(\rho, \eta)} \equiv 4 \beta_{2} \eta^{2} \sqrt{\rho}|V(2 \rho|\eta|)| e^{2 L_{2}(\rho, \eta)},
$$

несложно доказать первое из неравенств (44). Второе из них является следствием определения (29) функции $T_{2}$ и оценки (46) для функции $F_{\lambda}$. Соотношения (45) следуют из определения (29) функции $T_{1}$. Интеграл в этих соотношениях сходится на верхнем пределе, потому что $G_{\lambda}^{2}(t, \eta)=O(1)$ и $|V(2 t|\eta|)|=o\left(t^{-2}\right)$, если $t \rightarrow \infty$. Этим замечанием завершим доказательство леммы. 
Укажем важное следствие лемм 1-3: интегралы $B$ и $D$, использованные в п. 3.2 для редукции задач Коши (7), (8) и (7), (26) к системам интегральных уравнений (21) и (27), существуют при любых $\lambda \geqslant-1 / 2, \rho \geqslant 0$ и $b \geqslant 0$.

4.2. Теоремы существования и единственности. Представим план исследований, выполненных в настоящем пункте.

Последовательно докажем теоремы 1-3 существования и единственности непрерывных решений систем уравнений (21) и (27). Используем преимущества этих систем и одну и ту же схему, состоящую из трех этапов. На первом этапе методом последовательных приближений Пикара-Линделефа [20] построены формальные решения $y_{1}$ или $z_{1}$ первых уравнений системы $(21)$ или системы $(27)$ в виде бесконечных рядов $W$ или $X$. На втором этапе сначала использованы мажорантные оценки (34), (35), (39) и (44), полученные в леммах 1-3, и построены мажоранты модуля ряда $W$ или $X$ в виде гиперболических косинусов $\operatorname{ch} \tau, \operatorname{ch} \tau_{1}$ или $\operatorname{ch} \tau_{2}$, аргументами которых являются ограниченные функции $\tau, \tau_{1}$ или $\tau_{2}$ переменных $\rho$ и $\eta$. Затем с помощью таких мажорант и мажорантного признака Вейерштрасса [21] доказаны равномерная и абсолютная сходимость рядов $W$ или $X$ в классе непрерывных функций и тем самым установлено существование единственных и непрерывных решений $y_{1}=W$ или $z_{1}=X$ первых уравнений систем (21) или (27). На третьем и завершающем этапе найдены непрерывные решения $y_{2}$ и $z_{2}$ вторых уравнений этих систем как соответствующие интегральные образы (25) и (32) функций $y_{1}$ и $z_{1}$, представленных в виде рядов $W$ и $X$.

Теорему 1 докажем подробно, а теоремы 2 и 3 - схематически. Затем как следствие теорем 1-3 докажем итоговую теорему 4 об однозначной разрешимости задачи Коши (7), (8).

Начнем с наиболее простого случая $2 \lambda>-1$. Используем лемму 1.

Теорема 1. В случае $2 \lambda>-1$ система интегральных уравнений (21) на полуоси $\rho \geqslant 0$ имеет единственное решение $\left\{y_{1}, y_{2}\right\}$. Его компоненты $y_{1}$ и $y_{2}$ непреръвны на этой полуоси, а в пределе $\lambda \rightarrow \infty$ являются суммами

$$
y_{1}(\rho, \eta)=1+\varepsilon_{1}(\rho, \eta), \quad y_{2}(\rho, \eta)=\int_{0}^{\rho} P_{2}(t, \eta) d t+\varepsilon_{2}(\rho, \eta), \quad \rho \geqslant 0,
$$

в которых слагаемые $\varepsilon_{1}$ u $\varepsilon_{2}$ убывают не медленнее, чем $\lambda^{-1} u \lambda^{-3 / 2}$. В пределе $\rho \rightarrow 0$ компонента $y_{2}$ убъвает быстрее функиии $\rho^{2 \lambda+1}$.

ДокАЗАТЕЛЬство начнем с анализа первого уравнения системы (21). Применим метод последовательных приближений Пикара-Линделефа [20]. Определим все элементы $y_{1 m}(\rho, \eta)$ бесконечной итерационной последовательности $\left\{y_{1 m}\right\}_{m=0}^{\infty}$. Пусть $y_{10}(\rho, \eta) \equiv 1$ при любых $\rho$ и $\eta$, а каждый элемент $y_{1 m}(m=1,2, \ldots)$ есть правая часть первого уравнения системы $(21)$, в которой искомая компонента $y_{1}$ заменена элементом $y_{1, m-1}$ :

$$
\begin{aligned}
y_{10}(\rho, \eta) & \equiv 1, \\
y_{1 m}(\rho, \eta) & =1+\int_{0}^{\rho} P_{1}\left(\rho_{1}, \eta\right) d \rho_{1} \int_{0}^{\rho_{1}} P_{2}\left(\rho_{2}, \eta\right) y_{1, m-1}\left(\rho_{2}, \eta\right) d \rho_{2}, \quad m=1,2, \ldots .
\end{aligned}
$$


Следовательно, элемент $y_{1 m}$ является частичной суммой $W_{m}$ слагаемого $y_{10} \equiv 1$ и слагаемых $w_{n}$ с номерами $n=1, \ldots, m$,

$$
w_{n}(\rho, \eta) \equiv \int_{0}^{\rho} P_{1}\left(\rho_{1}, \eta\right) d \rho_{1} \int_{0}^{\rho_{1}} P_{2}\left(\rho_{2}, \eta\right) d \rho_{2} \ldots \int_{0}^{\rho_{2 n-1}} P_{1}\left(\rho_{2 n}, \eta\right) d \rho_{2 n},
$$

бесконечного ряда

$$
W(\rho, \eta)=1+\sum_{n=1}^{\infty} w_{n}(\rho, \eta)
$$

Поэтому итерационная последовательность $\left\{y_{1 m}\right\}_{m=0}^{\infty}$ имеет предел $y_{1, \infty}=W$, являющийся искомым решением $y_{1}$, тогда и только тогда, когда сходится ряд $W$.

Покажем, что ряд $W$ всегда сходится, причем абсолютно и равномерно. Начнем с оценки модуля каждого слагаемого $w_{n}, n \geqslant 1$, этого ряда. По определению (49) слагаемое $w_{n}$ - интеграл кратности $2 n$. Этот интеграл содержит произведение функций $P_{1}\left(\rho_{p}, \eta\right)$ и $P_{2}\left(\rho_{p+1}, \eta\right)$ с номерами $p=1,2, \ldots, 2 n-1$ и аргументами $\rho_{p}$ и $\rho_{p+1}$, удовлетворяющими неравенству $\rho_{p+1} \leqslant \rho_{p}$. Согласно определениям (33) и неравенству (34), доказанному в лемме 1 , модуль такого произведения не превосходит произведения производных $\partial_{\rho_{p}} \tau\left(\rho_{p}, \eta\right)$ и $\partial_{\rho_{p+1}} \tau\left(\rho_{p+1}, \eta\right)$, а функция $\tau(\rho, \eta)$ равна нулю в точке $\rho=0$. Поэтому из равенства (49) следует довольно простая мажорантная оценка, равномерная по обеим переменным $\rho, \eta$ :

$$
\begin{aligned}
\left|w_{n}(\rho, \eta)\right| & \leqslant \int_{0}^{\rho} \partial_{\rho_{1}} \tau\left(\rho_{1}, \eta\right) d \rho_{1} \int_{0}^{\rho_{1}} \partial_{\rho_{2}} \tau\left(\rho_{2}, \eta\right) d \rho_{2} \ldots \int_{0}^{\rho_{2 n-1}} \partial_{\rho_{2 n}} \tau\left(\rho_{2 n}, \eta\right) d \rho_{2 n}= \\
& =\frac{\tau^{2 n}(\rho, \eta)}{(2 n) !} .
\end{aligned}
$$

Используя ее и известное разложение гиперболического косинуса $\operatorname{ch} \tau$, получим следующую равномерную по обоим аргументам $\rho$ и $\eta$ оценку ряда (50):

$$
|W(\rho, \eta)| \leqslant 1+\sum_{n=1}^{\infty}\left|w_{n}(\rho, \eta)\right| \leqslant 1+\sum_{n=1}^{\infty} \frac{\tau^{2 n}(\rho, \eta)}{(2 n) !}=\operatorname{ch} \tau(\rho, \eta)<\infty, \quad \rho \geqslant 0 .
$$

Следовательно, функция $\operatorname{ch} \tau$, аргумент которой принимает только конечные значения, является ограниченной мажорантой ряда $W$. Поэтому на полуоси $\rho \geqslant 0$ ряд $W$ сходится равномерно и абсолютно по мажорантному признаку Вейерштраса [21].

Продолжим исследование ряда $W$. Оценка (51) означает, что при всех $n=1,2, \ldots$ интеграл в правой части равенства (49) сходится при любом $\rho \geqslant 0$. Следовательно, левая часть этого равенства, функция $w_{n}$, непрерывна при любых $n=1,2, \ldots$ и при всех $\rho \geqslant 0$.

Итак, на всей полуоси $\rho \geqslant 0$ ряд $W$ сходится абсолютно и равномерно, а все его слагаемые $w_{n}$ - непрерывные функции. Как известно из теории рядов [21], ряд обладающий такими свойствами, является непрерывной функцией на полуоси $\rho \geqslant 0$. В силу определений (49) и (50) слагаемых $w_{n}$ и их суммы $W$ эта сумма является решением $y_{1}=W$ первого уравнения системы $(21)$.

Покажем от противного, что это уравнение не имеет другого решения, непрерывного при $\rho \geqslant 0$. Пусть $\tilde{y}_{1}-$ еще одно решение. Тогда разность $\zeta=y_{1}-\tilde{y}_{1}$ удовлетворяет тождеству

$$
\zeta(\rho, \eta) \equiv \int_{0}^{\rho} P_{1}\left(\rho_{1}, \eta\right) d \rho_{1} \int_{0}^{\rho_{1}} P_{2}(t, \eta) \zeta(t, \eta) d t, \quad \rho \geqslant 0 .
$$


После $n \geqslant 2$ итераций это тождество благодаря равенству (49) примет вид

$$
\zeta(\rho, \eta) \equiv \int_{0}^{\rho} P_{1}\left(\rho_{1}, \eta\right) d \rho_{1} \int_{0}^{\rho_{1}} P_{2}(t, \eta) w_{n-1}(t, \eta) \zeta(t, \eta) d t, \quad n=2,3, \ldots, \quad \rho \geqslant 0 .
$$

Такое тождество и оценки $(34),(51)$ функций $P_{1} P_{2}$ и $w_{n-1}$ порождают неравенство

$$
|\zeta(\rho, \eta)| \leqslant \max _{0 \leqslant t<\rho}|\zeta(t, \eta)| \frac{\tau^{2 n}(\rho, \eta)}{(2 n) !}, \quad n=2,3, \ldots, \quad \rho \geqslant 0 .
$$

В пределе $n \rightarrow \infty$ это неравенство вырождается в соотношение $|\zeta(\rho, \eta)| \leqslant 0, \rho \geqslant 0$, из которого следует, что $y_{1}=\tilde{y}_{1}$ при любом $\rho \geqslant 0$. Поэтому первое уравнение системы (21) имеет только одно решение $y_{1}=W$.

Докажем первое из соотношений (47). Вследствие оценок (51) найденное решение $y_{1}=W$ на всей полуоси $\rho \geqslant 0$ удовлетворяет неравенству $\left|y_{1}(\rho, \eta)\right| \leqslant \operatorname{ch} \tau(\rho, \eta)$ и оценкам

$$
\begin{aligned}
\left|y_{1}(\rho, \eta)-y_{1 m}(\rho, \eta)\right| & \leqslant \sum_{n=m+1}^{\infty}\left|w_{n}(\rho, \eta)\right| \leqslant \\
& \leqslant \sum_{n=0}^{\infty} \frac{\tau^{2(n+m+1)}(\rho, \eta)}{(2 n+2 m+2) !}<\frac{\tau^{2(m+1)}(\rho, \eta)}{(2 m+2) !} \operatorname{ch} \tau(\rho, \eta) .
\end{aligned}
$$

Выведем важные следствия этих оценок. Заметим, что в пределе $\lambda \rightarrow \infty$ функция $\tau$ согласно ее определению (33) убывает, как $O\left(\lambda^{-1 / 2}\right)$. Поэтому из обсуждаемых оценок следует два вывода: в пределе $\lambda \rightarrow \infty$ при любом $m \geqslant 0$ функция $y_{1 m}$ является равномерной асимптотикой решения $y_{1}$, а в частном случае $m=0$ верно первое из соотношений (47).

Теперь построим и исследуем решение $y_{2}$ второго уравнения системы (21). Положив $y_{1}=W$ во втором из равенств $(25)$, получим интегральное представление

$$
y_{2}(\rho, \eta)=\int_{0}^{\rho} P_{2}(t, \eta) W(t, \eta) d t, \quad \rho \geqslant 0
$$

искомого решения $y_{2}$ через уже найденный ряд $W$. Введем последовательность $\left\{y_{2 m}\right\}_{m=0}^{\infty}$ с элементами

$$
y_{2 m}(\rho, \eta) \equiv \int_{0}^{\rho} P_{2}(t, \eta) y_{1 m}(t, \eta) d t, \quad m=0,1, \ldots
$$

Используя оценки (35) и $(51)$ для функций $P_{2}$ и $w_{n}$, убедимся в том, что на всей полуоси $\rho \geqslant 0$ найденное в виде интеграла решение $y_{2}$ непрерывно, ограничено,

$$
\begin{aligned}
\left|y_{2}(\rho, \eta)\right| & \leqslant \int_{0}^{\rho}\left|P_{2}(t, \eta)\right||W(t, \eta)| d t \leqslant \\
& \leqslant \max _{0 \leqslant t \leqslant \rho}\left|F_{\lambda}(t, \eta)\right| \int_{0}^{\rho} \partial_{t} \tau(t, \eta) \operatorname{ch} \tau(t, \eta) d t=\max _{0 \leqslant t \leqslant \rho}\left|F_{\lambda}(t, \eta)\right| \operatorname{sh} \tau(\rho, \eta)<\infty
\end{aligned}
$$


и удовлетворяет неравенствам

$$
\begin{aligned}
\left|y_{2}(\rho, \eta)-y_{2 m}(\rho, \eta)\right| & \leqslant \int_{0}^{\rho}\left|P_{2}(t, \eta)\right|\left|y_{1}(t, \eta)-y_{1 m}(t, \eta)\right| d t \leqslant \\
& \leqslant \max _{0 \leqslant t \leqslant \rho}\left|F_{\lambda}(t, \eta)\right| \sum_{n=0}^{\infty} \frac{\tau^{2(n+m+1)+1}(\rho, \eta)}{(2 n+2 m+3) !} \leqslant \\
& \leqslant \max _{0 \leqslant t \leqslant \rho}\left|F_{\lambda}(t, \eta)\right| \frac{\tau^{2(m+1)}(\rho, \eta)}{(2 m+2) !} \operatorname{sh} \tau(\rho, \eta) .
\end{aligned}
$$

Благодаря этим неравенствам в пределе $\lambda \rightarrow \infty$ при любом $m \geqslant 0$ функция $y_{2 m}$ является равномерной асимптотикой решения $y_{2}$, а в частном случае $m=0$ верно второе из соотношений (47).

Перейдем к доказательству того, что $y_{2}=o\left(\rho^{2 \lambda+1}\right)$ при $\rho \rightarrow 0$. Функция $y_{1}$ непрерывна в точке $\rho=0$ справа и равна единице в этой точке, следовательно, $y_{1} \sim 1+o(1)$ при $\rho \rightarrow 0$. Покажем, что этом же пределе функция $y_{2}$ быстро сходится к нулю. Для этого во втором из соотношений (25) положим $y_{1}=1+o(1)$, воспользуемся определением (22) функции $P_{2}$ и заменим функцию $F_{\lambda}$ ее асимптотикой $(13)$. Таким образом получим старшее слагаемое асимптотики функции $y_{2}$ в виде произведения,

$$
y_{2}(\rho, \eta) \sim-4 \eta^{2} C_{\lambda}^{2}(\eta) \int_{0}^{\rho} t^{2 \lambda+2} V(2 t|\eta|) d t, \quad \rho \rightarrow 0
$$

в котором интеграл убывает быстрее, чем $\rho^{2 \lambda+1}$. Действительно, применив правило Лопиталя [21], имеем

$$
\lim _{\rho \rightarrow+0} \rho^{-2 \lambda-1} \int_{0}^{\rho} t^{2 \lambda+2} V(2 t|\eta|) d t=\frac{1}{2 \lambda+1} \lim _{\rho \rightarrow+0} \rho^{2} V(2 \rho|\eta|)=0 .
$$

Следовательно, $y_{2}=o\left(\rho^{2 \lambda+1}\right)$ в пределе $\rho \rightarrow 0$, что и требовалось показать.

Осталось убедиться в том, что интеграл (52) - единственное решение второго уравнения системы $(21)$ в классе функций, непрерывных на полуоси $\rho \geqslant 0$. Предположим противное: пусть имеется еще одно решение $\tilde{y}_{2}$. Тогда согласно первому уравнению системы (25) функция $y_{1}=W$ является образом функции $y_{2}$, заданной как интеграл (52), и функции $\tilde{y}_{2}$ :

$$
W(\rho, \eta)=1+\int_{0}^{\rho} P_{1}(t, \eta) y_{2}(t, \eta) d t, \quad W(\rho, \eta)=1+\int_{0}^{\rho} P_{1}(t, \eta) \tilde{y}_{2}(t, \eta) d t, \quad \rho \geqslant 0 .
$$

Следовательно, разность $y_{2}-\tilde{y}_{2}$ удовлетворяет тождеству

$$
\int_{0}^{\rho} P_{1}(t, \eta)\left[y_{2}(t, \eta)-\tilde{y}_{2}(t, \eta)\right] d t \equiv 0, \quad \rho \geqslant 0 .
$$

После дифференцирования по аргументу $\rho$ это тождество становится соотношением $P_{1}\left(y_{2}-\tilde{y}_{2}\right) \equiv 0$, которое выполняется, только если $y_{2} \equiv \tilde{y}_{2}$ при $\rho \geqslant 0$. Следовательно, интеграл (52) - единственное непрерывное при $\rho \geqslant 0$ решение второго уравнения системы (21). Это утверждение завершает доказательство теоремы.

Приступим к анализу случая $2 \lambda=-1$. Последовательно докажем теоремы 2 и 3. 
Теорема 2. В случае $2 \lambda=-1$ система интегральных уравнений (21) имеет на отрезке $0 \leqslant \rho \leqslant b=0.05$ единственное решение $\left\{y_{1}, y_{2}\right\}$. Обе его компоненты $y_{1}$ и $y_{2}$ непрерывны на этом отрезке. В пределе $\rho \rightarrow 0$ компонента $y_{2}$ сходится к нулю быстрее функиии $1 / \ln \rho$.

ДокАЗАтЕльство. Предлагаемое доказательство отличается от доказательства теоремы 1 только тем, что вместо оценок (34) и (35) функций $P_{1} P_{2}$ и $P_{2}$ используются доказанные в лемме 2 оценки (39). Поэтому можно ограничиться лишь краткими пояснениями.

Для первого из уравнений (21) формулами (48) определим итерационную последовательность $\left\{y_{1 m}\right\}_{m=0}^{\infty}$. Ее предел является искомым решением $y_{1}$ и равен бесконечному ряду $W$, заданному равенствами (49) и (50). Благодаря оценке (39) любое слагаемое $w_{n}$ ряда $W$ ограничено:

$$
\left|w_{n}(\rho, \eta)\right| \leqslant \frac{\tau_{1}^{2 n}(\rho, \eta)}{(2 n) !}, \quad 0 \leqslant \rho \leqslant b .
$$

Поэтому $|W| \leqslant \operatorname{ch} \tau_{1}$ при $0 \leqslant \rho \leqslant b$. Следовательно, ряд $W$ сходится абсолютно и равномерно на всем отрезке $0 \leqslant \rho \leqslant b$ и на этом же отрезке является непрерывным решением $y_{1}=W$ первого уравнения системы (21). Второе из интегральных представлений (25), оценка (39) функции $P_{2}$ и неравенство $|W| \leqslant \operatorname{ch} \tau_{1}$ порождают следующие соотношения:

$$
y_{2}(\rho, \eta)=\int_{0}^{\rho} P_{2}(t, \eta) W(t, \eta) d t, \quad\left|y_{2}(\rho, \eta)\right| \leqslant \rho \operatorname{sh} \tau_{1}(\rho, \eta), \quad 0 \leqslant \rho \leqslant b .
$$

В силу этих соотношений на всем отрезке $0 \leqslant \rho \leqslant b$ компонента $y_{2}$ определяется однозначно и является непрерывной функцией.

Осталось исследовать поведение функции $y_{2}$ в пределе $\rho \rightarrow 0$. В этом пределе функция $y_{1}$ сходится к единице, а функция $F_{-1 / 2}$ имеет асимптотику (13). Поэтому из определения (22) функции $P_{2}$ и интегрального представления (25) следует соотношение

$$
y_{2}(\rho, \eta) \sim-4 \eta^{2} C_{-1 / 2}^{2}(\eta) \int_{0}^{\rho} t V(2 t|\eta|) d t, \quad \rho \rightarrow 0 .
$$

Сравним его правую часть с функцией $1 / \ln \rho$. Применив правило Лопиталя, получаем

$$
\lim _{\rho \rightarrow+0} \frac{1}{\ln \rho} \int_{0}^{\rho} t V(2 t|\eta|) d t=\lim _{\rho \rightarrow+0} \rho^{2} V(2 \rho|\eta|)=0 .
$$

Следовательно, в пределе $\rho \rightarrow 0$ функция $y_{2}$ сходится к нулю быстрее, чем $1 / \ln \rho$. Этим выводом закончим доказательство теоремы.

Перейдем к исследованию системы интегральных уравнений (27) для функций $z_{1}$ и $z_{2}$ на полуоси $\rho \geqslant b=0.05$. Используем лемму 3 .

Теорема 3. В случае $2 \lambda=-1$ и $b=0.05$ система интегральных уравнений (27) имеет на полуоси $\rho \geqslant b$ единственное непрерывное решение $\left\{z_{1}, z_{2}\right\}$.

ДокАзАтЕЛьство несущественно отличается от доказательства теоремы 1: вместо уравнений (21) и (25) и оценок (34) и (35) используем уравнения (27) и (32) и доказанные в лемме 3 оценки (44) и (45). Поясним лишь ключевые построения. 
Методом последовательных приближений построим формальное решение $z_{1}$ первого (для $i=1, j=2$ ) уравнения системы (27) в виде бесконечного ряда

$$
X(\rho, \eta)=z_{10}(\rho, \eta)+\sum_{n=1}^{\infty} x_{n}(\rho, \eta)
$$

со слагаемыми

$$
x_{n}(\rho, \eta) \equiv \int_{b}^{\rho} T_{1}\left(\rho_{1}, \eta\right) d \rho_{1} \int_{b}^{\rho_{1}} T_{2}\left(\rho_{2}, \eta\right) d \rho_{2} \ldots \int_{b}^{\rho_{2 n-1}} T_{1}\left(\rho_{2 n}, \eta\right) z_{10}\left(\rho_{2 n}, \eta\right) d \rho_{2 n} .
$$

Исследуем ряд $X$. Слагаемое $z_{10}$, как следует из его определения (28) и оценки (45), является всюду (при $\rho \geqslant b$ ) непрерывной и ограниченной функцией:

$$
\left|z_{10}(\rho, \eta)\right| \leqslant\left|z_{1}(b, \eta)\right|+\left|z_{2}(b, \eta)\right|\left|\int_{b}^{\rho} T_{1}(t, \eta) d t\right| \leqslant S_{0}(\eta) \equiv\left|z_{1}(b, \eta)\right|+\left|z_{2}(b, \eta)\right| p(\eta) .
$$

Построим мажорантную оценку $S_{n}$ слагаемого $x_{n}$ с номером $n \geqslant 1$. Сначала в интеграле (54) заменим все функции их модулями. Затем заменим $\left|z_{10}\right|$ функцией $S_{0}(\eta)$, а произведение функций $\left|T_{1}\left(\rho_{p}, \eta\right)\right|$ и $\left|T_{2}\left(\rho_{p+1}, \eta\right)\right|$ для каждого $p=1,2, \ldots, 2 n-1$ заменим превосходящим его согласно первому из неравенств (44) произведением производных $\partial_{\rho_{p}} \tau_{2}\left(\rho_{p}, \eta\right)$ и $\partial_{\rho_{p+1}} \tau\left(\rho_{p+1}, \eta\right)$. Используя равенство $\tau_{2}(b, \eta)=0$, вычислим полученный интеграл $S_{n}$ и запишем искомую оценку в следующем виде:

$$
\left|x_{n}(\rho, \eta)\right| \leqslant S_{n}(\rho, \eta) \equiv S_{0}(\eta) \frac{\tau_{2}^{2 n}(\rho, \eta)}{(2 n) !}<\infty, \quad n=1,2, \ldots, \quad \rho \geqslant b .
$$

Благодаря такой оценке модуль $|X|$ ряда (53) не превосходит произведения $S_{0} \operatorname{ch} \tau_{2}$ на всей полуоси $\rho \geqslant b$. Поэтому на той же полуоси этот ряд сходится абсолютно и равномерно и является единственным, причем непрерывным, решением $z_{1}=X$ первого (для $i=1, j=2$ ) уравнения системы (27).

Решение $z_{2}$ второго (для $i=2, j=1$ ) уравнения этой системы построим как образ (32) ряда (53) при $i=2, j=1$. Благодаря оценке (44) функции $T_{2}$ такой образ удовлетворяет неравенству

$$
\left|z_{2}(\rho, \eta)\right| \leqslant\left|z_{2}(b, \eta)\right|+S_{0}(\eta) \max _{b \leqslant t \leqslant \rho}\left|F_{\lambda}(t, \eta)\right| \operatorname{sh} \tau_{2}(\rho, \eta)<\infty, \quad \rho \geqslant b .
$$

Следовательно, полученное решение $z_{2}$ является однозначным и непрерывным на полуоси $\rho \geqslant b$. Этим выводом закончим доказательство теоремы.

Теперь, когда доказаны теоремы 1-3, нетрудно доказать итоговую теорему 4.

Теорема 4. При любом $\lambda \geqslant-1 / 2$ задача Коши (7), (8) однозначно разрешима в классе функиий, непрерывных на всей полуоси $\rho \geqslant 0$. Компоненты с и s решения $\{c, s\}$ этой задачи не имеют общих нулей, а компонента s удовлетворяет соотношению (14).

ДокАЗАТЕЛЬство. Используем теоремы 1-3 и представления (24) и (31).

В случае $2 \lambda>-1$ существование, единственность и непрерывность функций $c$ и $s$ на полуоси $\rho \geqslant 0$ следует из однозначности и непрерывности представлений (24) этих функций через функции $y_{1}$ и $y_{2}$ и теоремы 1 . Согласно этой же теореме в пределе 
$\rho \rightarrow 0$ компонента $y_{2}$ убывает быстрее функции $\rho^{2 \lambda+1}$. Благодаря этому свойству и представлениям (24) выполняется соотношение (14), обеспечивающее начальные условия (4) и (8) для волновой функции $u_{\lambda}$ и для амплитудных функций $c$ и $s$.

Анализ случая $2 \lambda=-1$ необходимо начать с определения значений функций $z_{i}(\rho, \eta), i=1,2$, в точке $\rho=b$. На отрезке $0 \leqslant \rho \leqslant b=0.05$ считаем функции $c$ и $s$ образами (24) функций $y_{1}$ и $y_{2}$. На полуоси $\rho \geqslant b$ для функций $c$ и $s$ используем их представления (31) через функции $z_{1}$ и $z_{2}$. Вследствие теоремы 2 и непрерывности отображений (24) обе функции $c$ и $s$ непрерывны на отрезке $0 \leqslant \rho \leqslant b$. В силу теоремы 3 и непрерывности отображений (31) функции $c$ и $s$ непрерывны на полуоси $\rho \geqslant b$ при любых конечных значениях $z_{i}(b, \eta)$ функций $z_{i}(\rho, \eta), i=1,2$, в точке $\rho=b$. Покажем, что функции $c$ и $s$ непрерывны и в точке $b$, если в равенствах (26) положить

$$
z_{i}(b, \eta)=y_{i}(b, \eta) \exp \left[(-1)^{i+1} B(b, \eta)\right], \quad i=1,2 .
$$

Согласно формулам (24)

$$
\lim _{\rho \rightarrow b-0} c(\rho, \eta)=y_{1}(b, \eta) e^{B(b, \eta)}, \quad \lim _{\rho \rightarrow b-0} s(\rho, \eta)=y_{2}(b, \eta) e^{-B(b, \eta)},
$$

а из равенств (31) следует, что

$$
\lim _{\rho \rightarrow b+0} c(\rho, \eta)=z_{1}(b, \eta), \quad \lim _{\rho \rightarrow b+0} s(\rho, \eta)=z_{2}(b, \eta) .
$$

Поэтому равенства (56) и только они обеспечивают непрерывность функций $c$ и $s$ в точке $\rho=b$. Далее считаем, что эти равенства выполняются. Используя теоремы 2 и 3 и дословно повторяя проведенный выше анализ случая $2 \lambda>-1$, доказываем все утверждения о свойствах функций $c$ и $s$ в случае $2 \lambda=-1$.

Осталось доказать, что при любом $\lambda \geqslant-1 / 2$ на полуоси $\rho \geqslant 0$ не существует точки, в которой обе функции $c$ и $s$ равны нулю. Ключевым для доказательства от противного будут два факта: функция $c$ непрерывна в точке $\rho=0$ и равна единице в этой точке.

Предположим противное: пусть $c(\rho, \eta)=0$ и $s(\rho, \eta)=0$ в точке $\rho=\infty$. Эти равенства используем как начальные условия для уравнений (7) в области $\rho>0$. Исследуем соответствующую задачу Коши. Подстановкой

$$
t=1-e^{-\rho}, \quad c(\rho, \eta)=\tilde{c}(t, \eta), \quad s(\rho, \eta)=\tilde{s}(t, \eta)
$$

сведем ее к линейной относительно неизвестных функций $\tilde{c}$ и $\tilde{s}$ задаче Коши на отрезке $0 \leqslant t \leqslant 1$. Запишем такую задачу в виде системы уравнений

$$
\partial_{t} \tilde{c}=S_{1}(t, \tilde{c}, \tilde{s}), \quad \partial_{t} \tilde{s}=S_{2}(t, \tilde{c}, \tilde{s}), \quad 0 \leqslant t \leqslant 1
$$

с тривиальными начальными условиями $\tilde{c}(t, \eta)=0$ и $\tilde{s}(t, \eta)=0$ в точке $t=1$.

На полуинтервале $0<t \leqslant 1$ функции $S_{1}$ и $S_{2}$ непрерывны по переменной $t$ и имеют непрерывные частные производные по переменным $\tilde{c}$ и $\tilde{s}$. Следовательно, выполнены оба достаточных условия теоремы Пикаро [20]. По этой теореме на полуинтервале $0<t \leqslant 1$ задача Коши для функций $\tilde{c}$ и $\tilde{s}$ имеет единственное и притом непрерывное решение. Очевидно, что им является тривиальное решение: $\tilde{c}(t, \eta) \equiv 0$ и $\tilde{s}(t, \eta) \equiv 0$ для всех $t \in(0,1]$. Так как $c(\rho, \eta)=\tilde{c}(t, \eta)$, мы получаем, что 
$c(\rho, \eta)=0$ при любом $\rho>0$, но $c(\rho, \eta)=1$ в точке $\rho=0$. Следовательно, в этой точке функция $c$ терпит разрыв первого рода, но по теореме 5 эта функция непрерывна в точке $\rho=0$ справа. Полученное противоречие означает, что ранее высказанное предположение о том, что $c(\infty, \eta)=0$ и $s(\infty, \eta)=0$, неверно.

Аналогичным образом нетрудно показать, что не существует конечного значения переменной $\rho$, при котором обе функции $c$ и $s$ обращаются в нуль.

Теорема полностью доказана.

\section{5. ПРИЛОЖЕНИЯ МЕТОДА}

Представим некоторые теоретически интересные приложения метода амплитудных функций. В п. 5.1 выведены приближение фазы рассеяния в пределе $\lambda \rightarrow \infty$. П. 5.2 посвящен формулировке и обоснованию нелинейной версии метода фазовых функций. В п. 5.3 обсуждается случай выключенного кулоновского потенциала $(\eta=0)$. В п. 5.4 исследовано трехмерное рассеяние $(\lambda=\ell$, где $\ell=0,1, \ldots)$.

5.1. Приближение фазы рассеяния при больших значениях индекса $\lambda$. Выведем и исследуем приближение фазы рассеяния $\delta_{\lambda}(\eta)$ в интересном с физической точки зрения пределе больших значений квантового числа $\lambda$. Для этого используем определение (23) интеграла $B$, представление (24) амплитудных функций $c$ и $s$ через функции $y_{1}$ и $y_{2}$ и установленные в теореме 1 равномерные асимптотики (47) функций $y_{1}$ и $y_{2}$.

В равенства (24) подставим вместо функций $y_{1}$ и $y_{2}$ их асимптотики (47). В результате получим равномерные (по $\rho \geqslant 0$ ) асимптотические представления амплитудных функций

$$
\begin{aligned}
& c(\rho, \eta)=\left[1+\varepsilon_{1}(\rho, \eta)\right] e^{B(\rho, \eta)}, \\
& s(\rho, \eta)=-\left[4 \eta^{2} \int_{0}^{\rho} V(2 t|\eta|) F_{\lambda}^{2}(t, \eta) d t+\varepsilon_{2}(\rho, \eta)\right] e^{-B(\rho, \eta)}, \quad \lambda \rightarrow \infty,
\end{aligned}
$$

где функции $\varepsilon_{1}$ и $\varepsilon_{2}$ убывают при любом $\rho \geqslant 0$ не медленнее, чем $O\left(\lambda^{-1 / 2}\right)$ и $O\left(\lambda^{-1}\right)$.

Заменив в представлениях (16)-(18) функции $c$ и $s$ их асимптотиками (57), можно получить приближения множителя $N(\eta)$, фазы $\delta_{\lambda}(\eta)$ и сечения $\sigma_{\lambda}(\eta)$ рассеяния в пределе $\lambda \rightarrow \infty$.

Для примера приведем приближение фазы рассеяния:

$$
\delta_{\lambda}(\eta) \approx-\left[4 \eta^{2} \int_{0}^{\infty} V(2 t|\eta|) F_{\lambda}^{2}(t, \eta) d t\right] \exp \left[-8 \eta^{2} \int_{0}^{\infty} V(2 t|\eta|) F_{\lambda}(t, \eta) G_{\lambda}(t, \eta) d t\right] .
$$

Упростим это приближение в случае потенциала $V(x)$, который убывает в пределе $x=2 t|\eta| \rightarrow \infty$ быстрее любой обратной степени аргумента $x$. Такой потенциал принято называть короткодействующим. Воспользуемся известным представлением [15], [16]

$$
F_{\lambda}(\rho, \eta)=C_{\lambda}(\eta) \rho^{\lambda+1} e^{i \rho} \Phi\left(a_{1}, a_{2} ;-2 i \rho\right), \quad a_{1}=\lambda+1+i \eta, \quad a_{2}=2 \lambda+2,
$$

и заметим, что в пределе $\lambda \rightarrow \infty$ модули обоих параметров $a_{1}$ и $a_{2}$ ряда Куммера $\Phi\left(a_{1}, a_{2} ;-2 i \rho\right)$ неограниченно возрастают. $\mathrm{K}$ сожалению, в данном случае асимптотика [22] ряда Куммера известна лишь на интервале $0 \leqslant \rho<\infty$. Использовав эту 
асимптотику, получим следующее представление:

$$
F_{\lambda}(\rho, \eta)=e^{-\pi \eta / 2} \sqrt{\frac{\lambda+1}{2 \lambda}}\left[\frac{e \rho}{2(\lambda+1)}\right]^{\lambda+1}\left[1+O\left(\lambda^{-1}\right)\right], \quad \lambda \rightarrow \infty, \quad \rho<\infty .
$$

Благодаря этому представлению из формулы (58) следует искомое приближение

$$
\delta_{\lambda}(\eta) \approx \frac{e^{-\pi \eta}}{2 \lambda}\left(\frac{e}{2}\right)^{2 \lambda+2}\left(\frac{1}{\lambda+1}\right)^{2 \lambda+1} \int_{0}^{\infty} t^{2 \lambda+2} V(2 t|\eta|) d t, \quad \lambda \rightarrow \infty,
$$

справедливое только в рассмотренном случае короткодействующего потенциала $V$. Упростить аппроксимацию (58) в других случаях не удалось из-за отсутствия равномерных по переменной $\rho$ асимптотик функций $F_{\lambda}$ и $G_{\lambda}$ в пределе $\lambda \rightarrow \infty$.

5.2. Нелинейная версия метода фазовых функций. Сформулируем и обоснуем нелинейную версию метода фазовых функций в теории двумерного рассеяния. Построим эту версию как редукцию предложенного выше метода амплитудных функций, основанного на задаче Коши (7), (8) и представлениях (11), (16) и (17).

Из представлений (16) и (17) получаем равенства

$$
\delta_{\lambda}(\rho, \eta)=\operatorname{arctg} \frac{s(\rho, \eta)}{c(\rho, \eta)}, \quad\left\{\begin{array}{l}
\cos \delta_{\lambda}(\rho, \eta) \\
\sin \delta_{\lambda}(\rho, \eta)
\end{array}\right\}=\frac{1}{\sqrt{c^{2}(\rho, \eta)+s^{2}(\rho, \eta)}}\left\{\begin{array}{c}
c(\rho, \eta) \\
s(\rho, \eta
\end{array}\right\} .
$$

Функцию $M(\rho, \eta)$ считаем равной функции $N^{-1}(\rho, \eta)$. Согласно формулам $(16)$

$$
M(\rho, \eta) \equiv N^{-1}(\rho, \eta)=\sqrt{c^{2}(\rho, \eta)+s^{2}(\rho, \eta)}
$$

Используя функции $\delta_{\lambda}$ и $M$, из представления волновой функции $u_{\lambda}$ в виде произведения (11) функции $U$ и нормировочного множителя (16) получаем представление

$$
u_{\lambda}(\rho, \eta)=\frac{M(\rho, \eta)}{M(\infty, \eta)}\left[\cos \delta_{\lambda}(\rho, \eta) F_{\lambda}(\rho, \eta)+\sin \delta_{\lambda}(\rho, \eta) G_{\lambda}(\rho, \eta)\right] .
$$

Теперь выведем уравнение для функции $\delta_{\lambda}$. Продифференцируем по переменной $\rho$ ее представление (59) через функции $c$ и $s$. В полученном соотношении заменим производные $\partial_{\rho} c$ и $\partial_{\rho} s$ правыми частями соответствующих уравнений $(7)$. Затем воспользуемся представлениями (59) косинуса и синуса функции $\delta_{\lambda}$ через функции $c$ и $s$. В итоге получим искомое нелинейное уравнение

$$
\partial_{\rho} \delta_{\lambda}(\rho, \eta)=-4 \eta^{2} V(2 \rho|\eta|)\left[\cos \delta_{\lambda}(\rho, \eta) F_{\lambda}(\rho, \eta)+\cos \delta_{\lambda}(\rho, \eta) G_{\lambda}(\rho, \eta)\right]^{2}, \quad \rho>0,
$$

с начальным условием $\delta_{\lambda}(\rho, \eta)=0$ при $\rho=0$.

Аналогичным образом, но дифференцируя тангенс $t_{\lambda}=s / c$ функции $\delta_{\lambda}$ или равенство (60), выведем на полуоси $\rho>0$ нелинейное уравнение

$$
\partial_{\rho} t_{\lambda}(\rho, \eta)=-4 \eta^{2} V(2 \rho|\eta|)\left[F_{\lambda}(\rho, \eta)+t_{\lambda}(\rho, \eta) G_{\lambda}(\rho, \eta)\right]^{2}
$$

с начальным условием $t_{\lambda}(\rho, \eta)=0$ при $\rho=0$ или линейное уравнение

$$
\partial_{\rho} M(\rho, \eta)=2 \eta^{2} V(2 \rho|\eta|) T_{\lambda}(\rho, \eta) M(\rho, \eta),
$$


где

$$
T_{\lambda}(\rho, \eta) \equiv 2 \cos 2 \delta_{\lambda}(\rho, \eta) F_{\lambda}(\rho, \eta) G_{\lambda}(\rho, \eta)+\sin 2 \delta_{\lambda}(\rho, \eta)\left[G_{\lambda}^{2}(\rho, \eta)-F_{\lambda}^{2}(\rho, \eta)\right],
$$

с начальным условием $M(\rho, \eta)=1$ при $\rho=0$. Такое уравнение имеет единственное решение:

$$
M(\rho, \eta)=\exp \left\{2 \eta^{2} \int_{0}^{\rho} V(2 t|\eta|) T_{\lambda}(t, \eta) d t\right\} .
$$

Как пояснялось в п. 3.2, функция $\delta_{\lambda}(\rho, \eta)$ называется фазовой и имеет прозрачный физический смысл. Из представления (61) следует, что $M\left(\rho_{d}, \eta\right) / M(\infty, \eta)$ является амплитудой, на которую волновая функция $u_{\lambda}$ отличается от множителя, заключенного в (61) в квадратные скобки. Поэтому функцию $M$ можно называть амплитудной функцией.

Итак, в предложенной выше нелинейной версии метода фазовых функций для полного решения краевой задачи Шредингера (3)-(5) необходимо сначала проинтегрировать нелинейное фазовое уравнение (62) или нелинейное уравнение (63), затем найти амплитудную функцию $M$ по формуле (64) и, наконец, вычислить волновую функцию $u_{\lambda}$ по формуле (61).

Сформулируем особо важный и новый результат как математическое обоснование нелинейной версии метода фазовых функций. Вследствие представления (59) фазовой функции $\delta_{\lambda}$ через амплитудные функции $c$ и $s$ и в силу однозначной разрешимости задачи Коши (7), (8) для этих функций фазовое уравнение (62) с начальным условием $\delta_{\lambda}=0$ при $\rho=0$ имеет единственное решение. Поэтому волновая и амплитудная функции $u_{\lambda}$ и $M$ определяются равенствами (61) и (64) однозначно. Интеграл в равенстве (64) существует при любом $\rho \geqslant 0$, если потенциал $V$ удовлетворяет условиям (2). В этом утверждении нетрудно убедиться, используя непрерывность и асимптотики (13) и (15) функций Кулона.

Завершим настоящий пункт самым простым доказательством утверждения о том, что функции $c$ и $s$ не имеют общих нулей. Используем функцию $M$. Согласно представлению (60) $M=\sqrt{c^{2}+s^{2}}$, а в силу представления (64) эта функция всегда положительна. Следовательно, при всех $\rho \geqslant 0$ верно неравенство $\sqrt{c^{2}+s^{2}}>0$, которое и означает, что функции $c$ и $s$ не имеют общих нулей.

5.3. Метод амплитудных функций в некулоновском пределе. Как известно [15], [16], в некулоновском пределе (при $|R| \rightarrow \infty, \eta \rightarrow 0$ ) функции Кулона $F_{\lambda}$ и $G_{\lambda}$ вырождаются в произведения $j_{\lambda}$ и $\tilde{n}_{\lambda}$ функции $\sqrt{\pi \rho / 2}$ и функций Бесселя $J_{m}$ и $Y_{m}$ целого порядка $m=\lambda+1 / 2$ :

$$
F_{\lambda}(\rho, \eta)=j_{\lambda}(\rho) \equiv \sqrt{\frac{\pi \rho}{2}} J_{m}(\rho), \quad G_{\lambda}(\rho, \eta)=-\tilde{n}_{\lambda}(\rho) \equiv-\sqrt{\frac{\pi \rho}{2}} Y_{m}(\rho)
$$

в пределе $\eta=0$. Следовательно, для перехода к некулоновскому пределу во всех соотношениях, приведенных в разделах 2-5, нужно выполнить три замены

$$
F_{\lambda}(\rho, \eta) \rightarrow j_{\lambda}(\rho), \quad G_{\lambda}(\rho, \eta) \rightarrow-\tilde{n}_{\lambda}(\rho), \quad 4 \eta^{2} V(2 \rho|\eta|) \rightarrow \frac{2 m_{1}}{(\hbar k)^{2}} \widetilde{V}\left(\frac{\rho}{k}\right),
$$

а затем положить $\eta=0$. В итоге краевая задача Шредингера (3)-(5) станет задачей для радиальной волновой функции $u_{\lambda}(\rho, 0)$ двумерного рассеяния квантовой 
частицы $p_{1}$ центральным потенциалом $\widetilde{V}(r), r=\rho / k$, а задача Коши $(7),(8)$ для амплитудных функций $c(\rho, 0)$ и $s(\rho, 0)$ сведется к системе уравнений

$$
\begin{aligned}
\partial_{\rho} c(\rho, 0) & =\frac{2 m_{1}}{(\hbar k)^{2}} \widetilde{V}\left(\frac{\rho}{k}\right)\left[c(\rho, 0) j_{\lambda}(\rho)+s(\rho, 0) \tilde{n}_{\lambda}(\rho)\right] \tilde{n}_{\lambda}(\rho), \\
\left.\partial_{\rho} s \rho, 0\right) & =-\frac{2 m_{1}}{(\hbar k)^{2}} \widetilde{V}\left(\frac{\rho}{k}\right)\left[c(\rho, 0) j_{\lambda}(\rho)+s(\rho, 0) \tilde{n}_{\lambda}(\rho, 0)\right] j_{\lambda}(\rho),
\end{aligned}
$$

с начальными условиями $c(\rho, 0)=1$ и $s(\rho, 0)=0$ при $\rho=0$.

Такие уравнения использовались ранее в работах [9] и [10]. В работе [9] вопрос о существовании и единственности решения $\{c(\rho, 0), s(\rho, 0)\}$ вовсе не обсуждался. В работе [10] лишь пояснялась схема ответа на этот вопрос. Дадим исчерпывающий ответ. Для этого заметим, что оценки (36), (38) и (40) функций Кулона оказались ключевыми для последовательного доказательства лемм 1-3 и теорем 1-4. Эти оценки верны при любом $\eta$, в том числе и при $\eta=0$. Таким образом, в этом случае все леммы 1-3 и теоремы 1-4 остаются в силе и являются математическим обоснованием метода амплитудных функций для двумерного рассеяния потенциалом $V$, удовлетворяющим условиям (2). В частности, теорема 4 гарантирует существование и единственность непрерывного решения системы уравнений (67) с начальными условиями $c=1$ и $s=0$ в точке $\rho=0$.

5.4. Метод амплитудных функций для трехмерного рассеяния. Поясним, как адаптировать предложенный метод амплитудных функций на случай трехмерного рассеяния суммой $V^{\mathrm{c}}+V$ двух потенциалов или одним потенциалом $V$.

В задаче (3)-(5) заменим полуцелый индекс $\lambda$ на целый индекс $\ell$ и положим $\ell=0,1, \ldots$. В результате получится задача Шредингера для радиальной компоненты $u_{\ell}$ волновой функции рассеяния квантовой частицы $p_{1}$ в трехмерном пространстве. Эта компонента определяет рассеяние суммой кулоновского и центрального потенциала $V$ в квантовом состоянии $|q, \ell\rangle$ с заданными значениями $q$ и $\ell$ волнового числа и углового момента.

При целом $\lambda=\ell$ случай $2 \lambda=-1$ не реализуется, поэтому леммы 2,3 и теоремы 2, 3 не потребуются. Оценки функций Кулона (36) и (38) целого порядка $(\lambda=\ell)$ перейдут в давно известные оценки Кларсфельда [23]. Следовательно, лемма 1 и теоремы 1, 4 остаются в силе и служат математическим обоснованием метода амплитудных функций для трехмерного рассеяния суперпозицией кулоновского потенциала и потенциала $V$, подчиненного соотношениям (2).

Теперь обсудим случай выключенного $(\eta=0)$ кулоновского потенциала. В этом случае согласно равенствам (65) функции Кулона целого порядка вырождаются в известные функции Риккати-Бесселя [18]. Следовательно, для перехода к обсуждаемому случаю во всех соотношениях, приведенных в разделах 2-5, нужно выполнить три замены (66), а затем положить $\lambda=\ell$ и $\eta=0$. После указанных преобразований ключевые оценки (36) и (38) выродятся в менее точные, чем давно известные оценки Мартина [24] для функции Риккати-Бесселя. Поэтому все утверждения, сформулированные в лемме 1 и теоремах 1 и 4, останутся верными и будут математическим обоснованием метода амплитудных функций для трехмерного рассеяния центральным потенциалом $V$, подчиненным соотношениям (2). 


\section{6. ЗАКЛЮЧЕНИЕ}

Перечислим и обсудим основные результаты настоящей работы.

Дана формулировка и математическое обоснование метода амплитудных функций, предназначенного для простого и исчерпывающего решения радиальной задачи $(3)-(5)$ двумерного $(2 \lambda=-1,1,3, \ldots)$ или трехмерного $(\lambda=\ell=0,1, \ldots)$ рассеяния квантовой частицы сумой кулоновского потенциала $V^{\mathrm{c}}=2 \eta / \rho$ и центрального потенциала $V$, удовлетворяющего довольно общим условиям (2).

Показано, что этот метод применим и для решения радиальной задачи (3)-(5) двумерного или трехмерного рассеяния в случае выключенного кулоновского потенциала $(\eta=0)$.

Из основных линейных уравнений (7) с условиями (8) выведены нелинейное фазовое уравнение (62) и представления (61) и (64) волновой и амплитудной функций $u_{\lambda}$ и $M$ через фазовую функцию $\delta_{\lambda}(\rho, \eta)$.

Сформулируем неоспоримые преимущества предложенного метода. В этом методе ключевыми являются амплитудные функции, удовлетворяющие довольно простой, а именно линейной, системе дифференциальных уравнений (7) первого порядка. Эта система сводится к совокупности двух не связанных с друг с другом интегральных уравнений (21) или (27), удобных для исследования различных свойств амплитудных функций.

В заключение отметим следующие факты. В известных монографиях [7], [19], посвященных методу фазовых функций в теории трехмерного рассеяния, существование и единственность фазовых и амплитудных функций лишь подразумевались. Доказанные в настоящей работе теоремы 1-4 являются существенным дополнением к этим монографиям и расширением метода фазовых функций на случай двумерного рассеяния.

\section{Список литературы}

[1] L. D. Caar, D. DeMille, R. V. Krems, J. Ye, "Cold and ultracold molecules: science, technology and applications", New J. Phys., 11:5 (2009), 055049, 87 pp.

[2] K. G. Nath, O. Ivasenko, J. M. MacLeod, J. A. Miwa, J. D. Wuest, A. Nanci, D. F. Perepichka, F. Rosei, "Crystal engineering in two dimensions: an approach to molecular nanopatterning", J. Phys. Chem. C, 111:45 (2007), 16996-17007.

[3] I. F. Silvera, J. T. M. Walraven, "Stabilization of atomic hydrogen at low temperature", Phys. Rev. Lett., 44:3 (1980), 164-168; "Spin-polarized atomic deuterium: stabilization, limitations on density, and adsorption energy on helium", 45:15 (1980), 1268-1271.

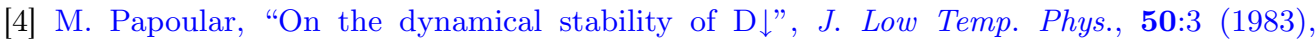
253-258.

[5] Ю. Каган, Г. В. Шляпников, И. А. Вартаньянц, Н. А. Глухов, “Квазидвумерный спинполяризованный атомарный водород", Писъма в ЖЖЭТФ, 35:9 (1982), 386-390.

[6] V.S. Melezhik, A. Negretti, "Confinement-induced resonances in ultracold atom-ion systems", Phys. Rev. A, 94:2 (2016), 022704, 8 pp., arXiv: 1602.05550.

[7] В. В. Бабиков, Метод фазовых функиий в квантовой механике, Наука, М., 1976.

[8] H. Friedrich, Scattering Theory, Lecture Notes in Physics, 872, Springer, Berlin, 2013.

[9] S. A. Rakityansky, N. Elander, "Analytic structure and power series expansion of the Jost function for the two-dimensional problem", J. Phys. A: Math. Theor., 45:13 (2012), 135209, $28 \mathrm{pp}$. 
[10] В. В. Пупышев, "Рассеяние медленной квантовой частицы аксиально-симметричным короткодействующим потенциалом", ЯФ, 77:5 (2014), 699-710.

[11] В. В. Пупышев, “Длина и эффективный радиус двумерного рассеяния квантовой частицы центральным короткодействующим потенциалом”, ТМФ, 180:3 (2014), 342-367.

[12] В. В. Пупышев, "Энергии слабосвязанных и околопороговых резонансных состояний квантовой частицы в двумерной плоскости", ТМФ, 179:1 (2014), 102-122; “Приближение эффективного радиуса в задаче двумерного рассеяния центральным короткодействующим потенциалом", 182:2 (2015), 315-337.

[13] G. Barton, "Rutherford scattering in two dimensions", Amer. J. Phys., 51:5 (1983), 420-422.

[14] D. Bollé, F. Gesztesy, "Scattering observables in arbitrary dimension $n \geqslant 2$ ", Phys. Rev. A, 30:3 (1984), 1279-1293.

[15] X. L. Yang, S. H. Guo, F. T. Chan, K. W. Wong, W. Y. Ching, "Analytic solution of a twodimensional hydrogen atom. I. Nonrelativistic theory", Phys. Rev. A., 43:3 (1991), 1186-1196.

[16] В. В. Пупышев, “Двумерное кулоновское рассеяние квантовой частицы: строение радиальных волновых функций”, ТМФ, 186:1 (2016), 123-141.

[17] В.В. Пупышев, "Двумерное кулоновское рассеяние квантовой частицы: волновые функции и функции Грина", ТМФ, 186:2 (2016), 252-271.

[18] Дж. Тейлор, Теория рассеяния. Квантовая теория нерелятивистских столкновений, Наука, М., 1975.

[19] Ф. Калоджеро, Метод фазовых функиий в теории потенциального рассеяния, Мир, M., 1972.

[20] Э. Камке, Справочник по обыкновенным дифференииальным уравнениям, Наука, М., 1976.

[21] Б. М. Будак, С. В. Фомин, Кратные интеграль и ряды, Физматлит, М., 2002.

[22] Г. Бейтмен, А. Эрдейи, Высшие трансцендентные функиии, т. 1: Гипергеометрическая функиия. Функиии Лежандра, Наука, М., 1973.

[23] S. Klarsfeld, "Behaviour of partial-wave amplitudes for large angular momenta in the presence of Coulomb forces", Nouvo Cimento A, 43:4 (1966), 1077-1094.

[24] A. Martin, "Some simple inequalities in scattering by complex potentials", Nouvo Cimento, 23:3 (1962), 641-654.

Поступила в редакцию 5.02.2016, после доработки 25.02.2016 